\title{
The Vibrio cholerae quorum-sensing protein VqmA integrates cell density, environmental, and host-derived cues into the control of virulence
}

Authors: Ameya A. Mashruwala ${ }^{1,2}$, Bonnie L. Bassler ${ }^{1,2 *}$

\author{
Affiliations: \\ ${ }^{1}$ Department of Molecular Biology, Princeton University, Princeton, New Jersey 08544 , \\ USA. \\ ${ }^{2}$ The Howard Hughes Medical Institute, Chevy Chase, MD 20815, USA. \\ *Correspondence to: bbassler@princeton.edu
}

\section{Scientific Abstract}

2 Quorum sensing is a chemical communication process in which bacteria use the 3 production, release, and detection of signal molecules called autoinducers to

4 orchestrate collective behaviors. The human pathogen Vibrio cholerae requires quorum

5 sensing to infect the small intestine. There, $V$. cholerae encounters the absence of oxygen and the presence of bile. We show that these two stimuli differentially affect quorum sensing function and, in turn, $V$. cholerae pathogenicity. The quorum-sensing receptor-transcription factor called VqmA, that detects the autoinducer called DPO, also detects the lack of oxygen and the presence of bile. Detection occurs via DPO-, oxygen, bile-, and redox-responsive disulfide bonds that alter VqmA DNA binding activity. We propose that VqmA serves as an information processing hub that integrates quorumsensing information, redox status, the presence or absence of oxygen, and host cues. In response to the information acquired through this mechanism, $V$. cholerae appropriately modulates its virulence output.

\section{Lay Abstract}

17 Quorum sensing (QS) is a process of chemical communication bacteria use to 18 orchestrate collective behaviors. QS communication relies on chemical signal molecules called autoinducers. QS regulates virulence in Vibrio cholerae, the causative agent of the disease cholera. Transit into the human small intestine, the site of cholera infection, exposes $V$. cholerae to the host environment. In this study, we show that the 
combination of two stimuli encountered in the small intestine, the absence of oxygen and the presence of host-produced bile, impinge on $V$. cholerae QS function and, in turn, pathogenicity. We suggest that possessing a QS system that is responsive to multiple environmental, host, and cell density cues enables $V$. cholerae to fine-tune its virulence capacity in the human intestine.

\section{Background:}

Quorum sensing (QS) is a process of cell-cell communication that bacteria use to synchronize group behaviors such as bioluminescence, DNA exchange, virulence factor production, and biofilm formation (20, 39, 45, 56). QS depends on the production, release, accumulation, and group-wide detection of extracellular signaling molecules called autoinducers $(\mathrm{Al})(45,53)$. At low cell density (LCD), when there are few cells present and the concentration of Als is low, the expression of genes driving individual behaviors occurs $(45,53,59)$. As the cells grow to high cell density (HCD), the extracellular concentration of Als likewise increases. Detection of accumulated Als drives the population-wide expression of genes required for group behaviors.

Vibrio cholerae is a Gram-negative enteric pathogen that causes infectious gastroenteritis. In $V$. cholerae, QS regulates collective behaviors including virulence factor production and biofilm formation $(27,39,66,67)$. Specifically, at LCD, genes encoding virulence factors and those required for biofilm formation are expressed (39). At HCD, genes required for both of these traits are repressed by QS (39). This pattern of gene expression is best understood in the context of the cholera disease. Infection is initiated by the ingestion of a small number of $V$. cholerae cells, and biofilm formation and virulence factor production are required for successful colonization $(66,67)$. In the host, the growth-dependent accumulation of Als launches the HCD QS program, which suppresses virulence factor production and biofilm formation, and triggers dispersal of the bacteria back into the environment. Indeed, V. cholerae strains "locked" into the LCD QS mode are more proficient in host colonization than strains "locked" in the HCD QS mode (27). Thus, QS is proposed to be crucial for $V$. cholerae transitions between environmental reservoirs and human hosts. 
V. cholerae produces and detects three Als, called AI-2, CAI-1and DPO (Figure 1) $(21,39,47,54)$. CAl-1 is used for intra-genus communication while Al-2 and DPO are employed for inter-species communication $(5,39,47)$. Different combinations of the three Als are thought to allow $V$. cholerae to distinguish the number of vibrio cells present relative to the total bacterial consortium. $V$. cholerae uses the information encoded in blends of Als to tailor its QS output depending on whether vibrios are in the minority or the majority of a mixed-species population $(5,39)$.

$\mathrm{Al}-2$ and $\mathrm{CAl}-1$ are detected by membrane-bound receptors that funnel information into a shared regulatory pathway (Figure 1) $(21,39)$. DPO is detected by the cytoplasmic VqmA receptor-transcription factor that activates expression of vqmR, encoding the VqmR regulatory small RNA (sRNA) $(34,46,47)$. Both Apo- and DPObound Holo-VqmA can activate vqmR expression with Holo-VqmA being more potent than Apo-VqmA (22). VqmR post-transcriptionally regulates target mRNAs (46). Important to this study is that at HCD, all three QS systems repress genes required for virulence and biofilm formation (Figure 1).

Upon the transition from the marine niche to the human host, $V$. cholerae switches from an aerobic to an anaerobic environment (15, 64). In addition, it encounters bile, which is abundant in the lower intestine, the primary site of $V$. cholerae infection. Bile is a heterogeneous mixture of compounds, including electrolytes and bile acids, and is estimated to be present at $\sim 0.2-2 \%$ weight/volume of intestinal contents (14). Bile is known to affect $V$. cholerae virulence gene expression by modulating activities of the oxidoreductase DsbA, the transmembrane-spanning transcription factor TcpP, and the ToxT transcription factor $(6,9,23,60,62,63)$. Bile also promotes biofilm formation in $V$. cholerae, and the second messenger molecule called cyclic-di-guanylate is involved in mediating this effect $(24,29)$.

Here, first we explore whether oxygen levels modulate QS in V. cholerae. We find that $V$. cholerae cultured under anaerobic conditions does not produce CAI-1, whereas increased DPO production does occur. In this work we focus on DPO. We show that the VqmA-DPO complex more strongly activates target gene expression

81 under anaerobic than aerobic conditions. One consequence of the presence/absence of oxygen is an altered reducing/oxidizing (hereafter, redox) cellular environment. We 
show that oxygen-dependent changes in VqmA activity are governed by cysteine disulfide bonds that are responsive to the redox environment. In the absence of DPO, during aerobic growth, Apo-VqmA forms an intra-molecular disulfide-bond that limits VqmA activity. By contrast, DPO-bound VqmA forms an inter-molecular disulfide-bond that enhances VqmA activity. The formation of the inter-molecular bond is not affected by oxygen levels. In the small intestine, $V$. cholerae encounters both the absence of oxygen and the presence of bile. Bile salts inhibit formation of the inter-molecular disulfide bond in VqmA. Thus, bile and DPO have opposing effects on VqmA-DPO activity. We propose that the VqmA-DPO-VqmR QS pathway allows $V$. cholerae to integrate QS information, host cues, and environmental stimuli into the control of genes required for transitions between the human host and the environment.

\section{Results:}

V. cholerae does not produce the CAI-1 QS Al under anaerobic conditions. To our knowledge, V. cholerae QS has only been studied under aerobic conditions. We know that the marine-human host lifecycle demands that $V$. cholerae transition between environments containing widely varying oxygen levels $(15,64)$. Moreover, QS is crucial in both $V$. cholerae habitats. Thus, we sought to investigate whether oxygen modulates $V$. cholerae QS. First, we assessed the relative levels of the three known QS Als from V. cholerae $\mathrm{C} 6706 \mathrm{Sm}^{\mathrm{R}}$ (hereafter wild-type; WT) following aerobic and anaerobic growth. Al activity in cell-free culture fluids was measured using a set of three bioluminescent $V$. cholerae strains, each of which exclusively reports on one QS AI (either Al-2, CAI-1, or DPO) when it is supplied exogenously.

Unlike $V$. cholerae cultured in the presence of oxygen (hereafter $+\mathrm{O}_{2}$ ), $V$. cholerae grown in the absence of oxygen (hereafter $-\mathrm{O}_{2}$ ) produced no CAI-1. Twice as much Al-2 and DPO accumulated in $V$. cholerae cultured $-\mathrm{O}_{2}$ than $+\mathrm{O}_{2}$ (Figure $2 \mathrm{~A}-\mathrm{C}$ ). We note that the dynamic ranges for the CAI-1 and DPO assay are $\sim 1,000-$ and $\sim 4$-fold, respectively, while that for the Al-2 assay is $\sim 100,000$-fold $(39,47)$. Thus, we consider the changes in CAl-1 and DPO to be physiologically relevant, whereas that for Al-2 is likely not, so we do not consider Al-2 further in this work. Additionally, V. cholerae cultured $-\mathrm{O}_{2}$ grew to a lower final cell density than when grown $+\mathrm{O}_{2}$ (Supplementary 
114 Figure 1A). We controlled for the reduced cell growth that occurs in the $-\mathrm{O}_{2}$ conditions, 115 nonetheless, no CAl-1 could be detected (Supplementary Figure 1B). Beyond lacking $\mathrm{O}_{2}$, our culture medium lacked an alternative terminal electron acceptor. Thus, we also considered the possibility that $V$. cholerae cultured in $-\mathrm{O}_{2}$ conditions was unable to respire and therefore unable to drive CAl-1 generation. However, supplementation of the $V$. cholerae $-\mathrm{O}_{2}$ cultures with the alternative terminal electron acceptor fumarate, which is readily consumed by $V$. cholerae (4), did not rescue CAl-1 production (Supplementary Figure 1B). Collectively, these data suggest that production of CAl-1 and DPO by $V$. cholerae is affected by oxygen levels. In the remainder of this study, we focus on the functioning of the DPO-VqmA QS circuit under different conditions that are predicted to be encountered in the host. We address possible ramifications of our results concerning $\mathrm{CAl}-1$ and $\mathrm{Al}-2$ in the Discussion.

VqmA exhibits increased activity in the absence of oxygen. Given that $V$. cholerae accumulated more DPO under $-\mathrm{O}_{2}$ conditions than $+\mathrm{O}_{2}$ conditions, we wondered whether the VqmA-DPO QS system would, in turn, display increased activity under $-\mathrm{O}_{2}$ conditions compared to $+\mathrm{O}_{2}$ conditions. VqmA controls the expression of the vqmR gene, encoding the small RNA VqmR (Figure 1). Therefore, expression of a vqmR-lacZ transcriptional fusion can be used to assess VqmA activity (47). Beta-galactosidase was selected as the reporter because its activity is not affected by oxygen. The vqmR-lacZ construct was integrated onto the chromosome of $\Delta t d h \mathrm{~V}$. cholerae. Tdh (threonine dehydrogenase) is required for DPO production (47). Thus, the $\Delta t d h$ strain makes no DPO but activates vqmR-lacZ expression in response to exogenously supplied DPO. We measured activity following growth in $+\mathrm{O}_{2}$ and $-\mathrm{O}_{2}$ conditions and in the absence and presence of exogenous DPO. In the presence of $\mathrm{O}_{2}$, vqmR-lacZ activity increased following supplementation with DPO (Supplementary

140 Figure 2A). Compared to the $+\mathrm{O}_{2}$ conditions, vqmR-lacZ activity was higher under $-\mathrm{O}_{2}$ 141 conditions, both in the absence and presence of DPO (Supplementary Figure 2A). 142 Increased DPO-independent vqmR-lacZ expression in the absence of $\mathrm{O}_{2}$ could be a 143 consequence of increased production of VqmA or increased VqmA activity. To 144 distinguish between these possibilities, we first examined whether changes in $\mathrm{O}_{2}$ levels 
alter VqmA production by quantifying VqmA-FLAG produced from the chromosome $1+\mathrm{O}_{2}$ and $-/+$ DPO. Similar levels of VqmA-FLAG were produced in all cases, suggesting that a change in VqmA abundance does not underlie increased vqmR-lacZ expression under $-\mathrm{O}_{2}$ conditions. (Supplementary Figure $2 \mathrm{~B}$ ). We next tested $\mathrm{O}_{2}$-driven changes in VqmA activity. To do this, we uncoupled expression of $v q m A-F L A G$ from its native promoter by cloning vqmA-FLAG onto a plasmid under an arabinose inducible promoter (hereafter, pvqmA-FLAG). We introduced the plasmid into a $\Delta v q m A \Delta t d h$. cholerae strain harboring the $v q m R-l a c Z$ chromosomal reporter and we measured both $\beta$ galactosidase output as well as VqmA-FLAG abundance in the same samples. VqmAFLAG levels did not change under the different conditions (Figure 3A), however, vqmRlacZ reporter activity normalized to cellular VqmA-FLAG levels increased in the cells exposed to DPO, and overall activity was $\sim 4-7$-fold higher under $-\mathrm{O}_{2}$ conditions than $+\mathrm{O}_{2}$ conditions both in the presence and absence of DPO (Figure 3B). We conclude that VqmA displays an increased capacity to activate gene expression under anaerobic conditions relative to aerobic conditions.

\section{VqmA forms intra- and inter-molecular disulfide bonds in an oxygen- and DPO-} dependent manner. We wondered what molecular mechanism drives the increase in VqmA activity under $-\mathrm{O}_{2}$ conditions (Figure 3). The cytoplasmic compartment of aerobically respiring $V$. cholerae is relatively oxidizing $(35,40)$. Thus, decreased oxygen levels would shift the cytoplasm to a reducing environment (42). Proteins can respond to such changes via redox-responsive cysteine residues $(35,38)$. Inspection of the VqmA amino acid sequence revealed the presence of four cysteine residues and all are strictly conserved in VqmA homologs in other vibrio species, but not in the VqmA receptor recently discovered in a vibriophage (Supplementary Figure 3 ). These findings led us to consider a model in which, in addition to activation by DPO, VqmA activity is regulated by redox-responsive cysteine residues.

Cysteine residues often undergo disulfide bond formation $(1,38,48)$. We assessed whether VqmA forms disulfide bonds in vivo and, if so, whether their formation is influenced by DPO and/or oxygen levels. We grew $\Delta v q m A \Delta t d h V$. cholerae carrying the pvqmA-FLAG construct under $+\mathrm{O}_{2}$ conditions. We subsequently divided the 
176 culture into four aliquots. One portion was untreated $\left(+\mathrm{O}_{2}\right.$, -DPO), one portion was

177 supplied DPO $\left(+\mathrm{O}_{2},+\mathrm{DPO}\right)$, one portion was deprived of oxygen $\left(-\mathrm{O}_{2},-\mathrm{DPO}\right)$, and one 178 portion was deprived of oxygen and supplemented with DPO $\left(-\mathrm{O}_{2},+\mathrm{DPO}\right)$. We 179 extracted protein and analyzed the VqmA-FLAG protein profiles by immunoblot. These analyses were performed with or without the addition of the reductant $\beta$ mercaptoethanol (BME) to distinguish between VqmA-FLAG species that had and had not formed disulfide bonds. Previous studies have shown that the presence of an intramolecular disulfide bond leads to a protein species displaying increased gel mobility compared to the same protein lacking the bond $(2,55)$. By contrast, inter-molecular disulfide bonds produce cross-linked protein oligomers that migrate with slower mobility than the corresponding monomers $(16,44)$. We first consider the results for VqmA under $+\mathrm{O}_{2}$ conditions: Under non-reducing conditions (-BME) and in the absence of DPO, VqmA-FLAG displayed mobility consistent with an oxidized monomer (labeled OMonomer; Figure 4A and 4B lane 1). Treatment with BME caused VqmA-FLAG to migrate more slowly, consistent with it being a reduced monomer (labeled R-Monomer; Figure $4 \mathrm{~A}$ and $4 \mathrm{~B}$ lane 2). Administration of DPO drove formation of an additional VqmA-FLAG species, corresponding in size to an oxidized dimer (labeled O-Dimer, 193 Figure 4A and 4B lane 3), but only under oxidizing (i.e., -BME) conditions (Figure 4B, compare lanes 3 and 4). These results suggest that, under aerobic conditions, a fraction of VqmA harbors an intra-molecular disulfide bond and DPO-bound VqmA forms an inter-molecular disulfide bond. Under anaerobic conditions, the portion of VqmA containing the intra-molecular disulfide bond decreased (Figure 4B compare lane 1 to lane 5 and lane 5 to lane 6 ) while the DPO-dependent inter-molecular disulfide bonded species was unaffected by the absence of oxygen (Figure 4A compare lane 3 to lane 7 and lane 7 to lane 8).

To garner additional evidence for the presence of an intra-molecular disulfide bond(s) in the species designated VqmA O-monomer in Figure 4B, we treated samples prepared from cells grown under $+\mathrm{O}_{2}$ and -DPO conditions with methoxypolyethylene glycol maleimide (m-MAL-PEG) (32). m-MAL-PEG alkylates reduced cysteine residues 205 and in so doing, confers an $\sim 5 \mathrm{kDa}$ molecular weight change for each alkylation event 206 (see schematic in Figure 4A). VqmA contains four cysteine residues, thus the fully 
reduced protein would undergo $4 \mathrm{~m}$-MAL-PEG events while if one intra-molecular disulfide bond exists, only two cysteine residues could react. Following treatment, ApoVqmA-FLAG migrated primarily as two bands, corresponding to four ( $40 \%$ of protein) and two ( $60 \%$ of protein) alkylation events (Figure $4 \mathrm{C}$, compare lane 1 to lane 2), confirming that while a portion of VqmA-FLAG is fully reduced in vivo, the majority of the protein exists as an oxidized species containing one intra-molecular disulfide bond.

To determine the residues involved in VqmA disulfide linkages, we conducted two experiments. First, we employed an in vitro thiol-trapping strategy based on sequential reactions that modify accessible cysteine residues with two thiol-specific reagents (Supplementary Figure 4A) (52). In the initial thiol-blocking step, purified 6HisVqmA treated with diamide, a thiol-specific oxidant (30), was denatured and incubated with chloroacetamide (CAA). CAA alkylates accessible cysteine residues (i.e., those not involved in disulfide bonds), blocking them from further modification. The CAA treated sample was next treated with TCEP, a reductant, enabling non-CAA labeled cysteines to be reduced. These newly freed residues were labeled in the final alkylation step with $\mathrm{N}$-ethylmaleimide (NEM). The sample was then analyzed by mass spectrometry. The logic is that if a particular cysteine residue was inaccessible to CAA due to disulfide bonding, it would be preferentially labeled with NEM in the subsequent NEM modification step. Thus, the NEM/CAA ratio would be $>1$. By contrast if a cysteine residue was not involved in disulfide modification, it would be preferentially labeled with CAA and have a NEM/CAA ratio < 1. The VqmA C48 and C63 residues had NEM/CAA ratios of $\sim 100$ and $\sim 10$, respectively, suggesting both of these residues are involved in disulfide-linkages (Supplementary Figure 4B). We were unable to obtain good coverage of the C134 and C22 residues using this technique so we could not similarly assess them.

Our second experiment to probe disulfide bonds in VqmA relied on mutagenesis. We individually substituted an alanine residue for each cysteine residue in the pvqmAFLAG construct, introduced the plasmids into the $\Delta v q m A \Delta t d h \mathrm{~V}$. cholerae strain, and repeated the analyses described in Figure 4C. We first consider the case of intramolecular disulfide bond formation under $+\mathrm{O}_{2}-\mathrm{DPO}$ conditions. In the mutant proteins, following replacement of a cysteine residue with alanine, a maximum of three residues 
can react with $m-M A L-P E G$ in the fully reduced protein (Figure $4 A$, top). However, if an intra-molecular disulfide bond is present, then only one cysteine residue can be decorated with m-MAL-PEG. Figure 4C shows that the Apo-VqmA C22A and ApoVqmA C134A proteins each migrated as two bands, a result consistent with portions of each protein harboring one and three m-MAL-PEG moieties (Figure 4C; compare lane 3 to lanes 2 and 1; and lane 6 to lanes 2 and 1). This result suggests that the fraction of Apo-VqmA C22A and the fraction of Apo-VqmA C134A that exhibit one m-MAL-PEG decoration harbor intra-molecular disulfide bonds. Apo-VqmA C48A and Apo-VqmA C63A migrated largely as single bands at the region corresponding to three m-MALPEG decorations (Figure 4C; compare lanes 4 and 5 to lane 2), suggesting that these proteins exist as reduced species and are thus incapable of forming intra-molecular disulfide bonds. Therefore, we conclude that in WT VqmA, an intra-molecular disulfide bond is formed between cysteine residues 48-63.

Next, we consider inter-molecular disulfide bond formation under $+\mathrm{O}_{2}+\mathrm{DPO}$ conditions, and under non-reducing conditions (i.e., -BME). Figure 4D shows that the Holo-VqmA C22A, Holo-VqmA C48A, and Holo-VqmA C63A proteins migrated as mixtures of oxidized monomers and oxidized dimers, while the Holo-VqmA C134A protein migrated exclusively as an oxidized monomer (compare lanes 2-5 to lane 1). These data suggest that in Holo-VqmA, there is a C134-C134 inter-molecular disulfide linkage. We also constructed and assessed the double VqmA C63A C134A mutant under $+\mathrm{O}_{2}+\mathrm{m}-\mathrm{MAL}-\mathrm{PEG}$ and $+\mathrm{O}_{2}+\mathrm{DPO}$ conditions. Figure $4 \mathrm{C}$ shows that under aerobic conditions, all of the Apo-VqmA C63A C134A protein contains two m-MAL-PEG decorations (compare lane 7 to lane 2), confirming that the two remaining cysteine residues were accessible and that the protein is fully reduced. Figure 4D shows that under aerobic conditions Holo-VqmA C63A C134A migrates entirely as a monomer (compare lanes 6 to lane 1). Thus, Holo-VqmA C63A C134A is incapable of forming both intra- and inter-molecular disulfide bonds.

Collectively our data suggest that: 1) VqmA forms disulfide bonds in vivo and in vitro; 2) an intra-molecular disulfide bond is formed between VqmA C48-C63, and an inter-molecular disulfide bond is made between C134-C134, and 3) disulfide bond formation is influenced by both oxygen and DPO. 
VqmA activity is limited by the intra-molecular disulfide bond and enhanced by the inter-molecular disulfide bond. To explore the in vivo consequences of VqmA disulfide bond formation on VqmA function, we tested whether the Apo- and Holomutant VqmA proteins that are incapable of forming particular intra- and/or intermolecular disulfide bonds displayed altered abilities to activate target vqmR transcription (see schematic in Supplementary Figure $5 A$ ). We introduced the vqmAFLAG, vqmA C48A-FLAG and the vqmA C134A-FLAG alleles onto the chromosome of a $V$. cholerae $\Delta t d h$ strain carrying $v q m R-l a c Z$ and measured reporter activity following aerobic growth +/- DPO. Our rationale was that WT VqmA forms both the C48-C63 intra-molecular bond and the C134-C134 inter-molecular bond. By contrast the VqmA C48A variant is unable to form the C48-C63 intra-molecular bond and the VqmA C134A variant is unable to form the C134-C134 inter-molecular bond (from Figure 4C and 4D). Therefore, by comparing the activities of these three proteins, we could assess the effect of individually eliminating each disulfide bond on VqmA activity. We likewise made a strain carrying vqmA C63A C134A-FLAG on the chromosome to examine the effect of simultaneous elimination of both disulfide bonds.

First we consider the case of Apo-VqmA. Apo-VqmA C48A and Apo-VqmA C134A exhibited an $\sim 2$-fold and $\sim 4$-fold increase and decrease, respectively, in reporter activity, relative to WT Apo-VqmA (Supplementary Figure S5B). Apo-VqmA C63A C134A exhibited a $\sim 3$-fold increase in reporter activity relative to WT Apo-VqmA and a $\sim 10$-fold increase in reporter activity relative to the strain carrying the Apo-VqmA C134A single mutant (Supplementary Figure S5B). Thus, we conclude that the C48-C63 intramolecular disulfide bond limits transcriptional activity of Apo-VqmA.

Next, we consider the case for Holo-VqmA. In cultures supplemented with DPO, vqmR-lacZ reporter activity increased $\sim 20$-30-fold, in a DPO-concentration-dependent 294 manner in the strain carrying WT Holo-VqmA relative to the strain with WT Apo-VqmA 295 (Supplementary Figure S5C). The strain carrying the Holo-VqmA C48A variant displayed a further increase in DPO-dependent reporter activity relative to the WT Holo-

297 VqmA. However, in strains harboring Holo-VqmA C134A and Holo-VqmA C63A C134A, 298 only modest (3-4-fold) responses to DPO occurred (Supplementary Figure S5C). Thus, 
the DPO-responsive, $\mathrm{C} 134-\mathrm{C} 134$ inter-molecular disulfide-bond enhances VqmA transcriptional activation activity.

\section{VqmA activity is differentially modulated by the cellular redox environment. To} test whether VqmA activity is responsive to cellular redox, we supplemented the strains carrying the different VqmA variants with DTT, a cell-permeable reductant. We reasoned that if the absence of oxygen generates a reducing environment that prevents the formation of a particular disulfide bond, addition of DTT would mimic this condition by promoting a reducing cellular environment, including in the presence of oxygen. To control for any potential DTT-induced changes in the levels of chromosomally expressed $v q m A$, we introduced plasmids harboring arabinose inducible vqmA-FLAG, vqmA C134A-FLAG, or vqmA C63A C134A-FLAG into the $V$. cholerae $\triangle$ vqmA $\Delta t d h$ strain carrying vqmR-lacZ and measured reporter activity +/- DPO and +/- DTT.

In the absence of DTT, the Apo- and Holo- plasmid-borne VqmA variants displayed reporter activities similar to when the variants were expressed from the chromosome (plasmid-borne variants are in Figure 5A and 5B, compare the black bars in each panel; for chromosomal variants, see Figure S5B and C). Treatment with DTT increased reporter activity for WT Apo-VqmA and Apo-VqmA C134A but did not alter the reporter activity in the strain carrying Apo-VqmA C63A C134A (Figure 5A, compare black and gray bars for each strain). As a reminder, WT Apo-VqmA and Apo-VqmA C134A form the C48-C63 intra-molecular disulfide bond, while the Apo-VqmA C63A C134A protein does not. Thus, these data suggest that a reducing environment interferes with formation of the C48-C63 intra-molecular disulfide bond, thereby eliminating its negative effect on Apo-VqmA activity.

Regarding WT Holo-VqmA, reporter activity diminished by $\sim 6$-fold when DTT was present in addition to DPO (Figure 5B; compare the first pair of black and gray bars). In contrast, DTT supplementation did not significantly affect reporter activity in the strain carrying Holo-VqmA C134A (Figure 5B; compare the second set of black and gray bars), while $\sim 3$-fold lower activity was produced by the strain carrying Holo-VqmA C63A C134A (Figure 5B; compare the third set of black and gray bars). Since the activity of VqmA C134A was not affected by DTT supplementation, we conclude that the C134- 
C134 inter-molecular bond does not form in a reducing environment, and without that bond, Holo-VqmA transcriptional activity is diminished.

VqmA DNA binding capacity is differentially modulated by its redox environment. We suspected that the in vivo redox-dependent changes in VqmA disulfide bond formation would have ramifications on VqmA DNA binding capability. To explore this notion, we examined the ability of purified VqmA proteins to bind pvqmR promoter DNA. First, to examine the role of the C48-C63 intra-molecular disulfide bond, we assessed DNA binding for 6His-VqmA C134A treated with diamide (to enable disulfide bond formation) or DTT (to prevent disulfide bond formation) (see Supplementary Figure 6). Relative to the DTT treated protein, the diamide treated protein exhibited a 4-fold reduction in DNA binding (Figure 5C; compare lanes 2-5 with lanes 7-10). This result suggests that formation of the C48-C63 intra-molecular disulfide bond limits DNA binding.

We investigated the role of the VqmA C134-C134 inter-molecular disulfide bond on DNA binding by comparing the DNA binding capabilities of $6 \mathrm{His}-\mathrm{VqmA}$ and $6 \mathrm{His}-$ VqmA C134A. Diamide treated 6His-VqmA was approximately twice as potent at binding DNA as was 6His-VqmA C134A [compare left halves of Figure 5C (VqmA C134A) and Figure 5D (WT VqmA)], suggesting that the C134-C134 inter-molecular disulfide bond promotes DNA binding. The DTT treated 6His-VqmA C134A and 6HisVqmA proteins showed no difference in DNA binding capability [compare right halves of Figure 5C (VqmA C134A) and Figure 5D (WT VqmA)]. Like 6His-VqmA C134A, DTT treated 6His-VqmA was more proficient in DNA binding than diamide treated 6HisVqmA, consistent with intra-molecular disulfide bond formation limiting DNA binding (Figure 5D). Collectively, the data in Figures 4 and 5 suggest a model in which the transcriptional and DNA binding activities of both Apo-VqmA and Holo-VqmA are modulated by disulfide bond formation, the cytoplasmic redox environment, and by the level of $\mathrm{O}_{2}$ in the environment. 
signal transduction pathway, which represses virulence factor production and biofilm formation, is most highly active under anaerobic conditions. $V$. cholerae encounters anaerobiosis in the human intestine. The paradox is that in the intestine, $V$. cholerae is virulent and makes biofilms. We thus wondered if a possible host intestinal signal(s) could modulate VqmA-DPO signaling, allowing infection to proceed under anaerobic conditions. Bile salts, present in high concentrations in the human small intestine, can alter the redox environment of bacterial cells and thereby affect disulfide bond formation in cytoplasmic proteins (11). Thus, we were inspired to investigate whether bile salts could abrogate VqmA-DPO transcriptional activation activity. We cultured the $\triangle v q m A$ $\Delta t d h V$. cholerae strain carrying the arabinose inducible pvqmA-FLAG construct and $v q m R$-lacZ on the chromosome in the presence and absence of oxygen, bile, and DPO and we measured reporter activity. Bile treatment caused $\sim 2$ - fold and $\sim 10$-fold decreases in vqmR-lac $Z$ reporter activity under $+\mathrm{O}_{2}$ and $-\mathrm{O}_{2}$ growth, respectively (Figure 6A; first four bars). Bile supplementation also decreased vqmR-lacZ reporter activity in cultures supplied with DPO, again with the maximum effect observed under $\mathrm{O}_{2}$ growth (Figure $6 \mathrm{~A} ; 5^{\text {th }}$ bar onward). To test whether the presence of bile affects VqmA disulfide bonds, we used analyses similar to those in Figure 4B. Consistent with the vqmR-lacZ reporter activity, bile supplementation prevented formation of O-dimers, both in the presence and absence of $\mathrm{O}_{2}$, suggesting it interferes with the Holo-VqmA C134-C134 inter-molecular disulfide bond (Figure 6B).

\section{Bile salt-mediated disruption of VqmA-DPO-driven signal transduction promotes}

V. cholerae virulence. In V. cholerae, VqmA-DPO-directed production of VqmR results in decreased expression of genes involved in biofilm formation and virulence factor expression, including vpsL and $t c p A$, respectively $(46,47)$. VpsL is required to synthesize $V$. cholerae exopolysaccharide, an essential component of the biofilm matrix, and TcpA is a virulence factor required for $V$. cholerae to colonize the human small intestine $(17,57)$. Our finding that bile supplementation inhibited VqmA-DPO function and that the effect of bile-mediated inhibition occurred primarily in the absence of oxygen, led us to predict that the repression of $v p s L$ and $t c p A$ expression would also be maximally disrupted following bile supplementation in the absence of oxygen. We 
measured transcript levels of $v p s L$ and tcpA in the WT and $\triangle v q m A$ strains following exposure to $25 \mu \mathrm{M}$ DPO, bile, deprivation of oxygen, or combinations of the three treatments.

In WT $V$. cholerae, bile supplementation modestly increased vpsL and tcpA transcript levels ( $\sim 3-$ and $\sim 2$-fold, respectively) however, only under $-\mathrm{O}_{2}-\mathrm{DPO}$ conditions (Supplementary Figure 7 and Figure 6C). These data suggest that bile induces genes required for biofilm formation and virulence in the absence of oxygen and Holo-VqmA can override the effect of bile. Transcript levels for both $v p s L$ and $t c p A$, under $-\mathrm{O}_{2}-$ DPO conditions were further increased in the $\triangle v q m A$ strain treated with bile ( $\sim 6$ - and $\sim 3$-fold, respectively). We interpret this result to mean that bile also induces an increase in $v p s L$ and $t c p A$ expression through a pathway that does not involve VqmA. However, because the major effect of bile occurs only in the $\Delta v q m A$ strain, we conclude that this additional pathway is epistatic to VqmA in the control of $v p s L$ and tcpA.

We tested whether the above changes in gene expression translated into alterations in $V$. cholerae pathogenicity by assaying whether bile affected cytotoxicity of $V$. cholerae in co-culture with human Caco-2 intestinal cells. We generated differentiated monolayers of Caco-2 cells and co-cultured them with either WT or $\Delta v q m A V$. cholerae in the presence and absence of bile. The presence of bile, increased $V$. cholerae-mediated cytotoxicity to Caco-2 cells with the $\triangle v q m A$ strain driving twice as much killing as WT V. cholerae (Figure 6D). Collectively, our data suggest that bile salts induce increased $V$. cholerae virulence when bacteria are deprived of oxygen, part of the bile effect is exerted via interference with the VqmADPO-VqmR QS circuit, and the presence of VqmA limits the ability of bile to affect target gene expression.

\section{Discussion:}

The diversity of environments that $V$. cholerae inhabits, from the ocean to marine organisms to the human stomach to the human intestine, necessitates that the bacterium rapidly perceives changes in its external environment and appropriately tailors its gene expression programs. Our current study reveals that $V$. cholerae alters both which Als are produced and the functioning of the VqmA-DPO-VqmR QS circuit in 
response to its environment. To our knowledge, these findings represent the first dissection of QS activity in the absence of oxygen in a facultative aerobic bacterium. We find that 1 ) the amount of an $\mathrm{Al}(\mathrm{CAI}-1)$ produced is dictated by oxygen levels; 2$)$ a single QS protein (VqmA) is capable of integrating information from three sources ( $\mathrm{Al}$, oxygen, bile); and 3) two disulfide bonds in a QS receptor (VqmA) antagonize one another with respect to their effects on protein activity, thereby aiding in perception and response to changes in cellular redox.

The benefit(s) of producing and detecting multiple QS Als has long been mysterious with respect to $V$. cholerae biology. Evidence suggests that each Al conveys specific information into the cell: CAl-1 measures the abundance of vibrios (kin) and Al2 and DPO measure the level of non-vibrio (non-kin) in the vicinity $(5,46,47)$. Our finding that CAl-1 is not produced in the absence of oxygen suggests that CAl-1 may also convey information about the external environment. Strains lacking the ability to synthesize CAl-1 display reduced survival in seawater and following challenge with oxidative stress (25). Thus, we propose that CAl-1 could drive the expression of genes required for the aerobic segment of the $V$. cholerae lifecycle and we are now testing this idea. The fact that CAl-1 is not produced under anoxia suggests that $V$. cholerae cannot take a census of kin in the absence of oxygen. Either kin counting is dispensable under anoxia or, perhaps, another molecule(s)/mechanism performs this function. By contrast, at a minimum based on sequencing data, thousands of bacterial species, including those found in the human microbiota, can synthesize Al-2, the Al used for inter-species communication (49). We found that Al-2 is synthesized in the absence of oxygen. Perhaps measurement of the abundance of non-kin bacteria is of paramount importance in densely populated niches containing complex bacterial consortia. Biosynthesis of both $\mathrm{Al}-2$ and $\mathrm{CAl}-1$ requires $S$-adenosylmethionine (SAM), an abundant metabolite that is crucial for methylation reactions $(8,28,54)$. Thus, another possibility is that in $\mathrm{V}$. cholerae, during periods of SAM limitation, CAI-1 production is curbed as a means of sparing SAM for other uses. Continued Al-2 production could suffice for QS-mediated cell-density tracking. Moreover, when SAM is used to produce $\mathrm{Al}-2$, but not CAI-1, SAM is regenerated via downstream reactions $(54,61)$. Thus, making Al-2 from SAM would not deplete the SAM reservoir. 
Oxygen is a terminal electron acceptor and therefore a critical substrate for bacterial growth. The human intestine is devoid of oxygen, and invading bacteria, such as $V$. cholerae that normally inhabit the relatively oxygenated marine environment, need to alter their physiology to survive. With the exception of a handful of studies (31, 33, 35), the molecular mechanisms by which $V$. cholerae perceives the absence of oxygen, and translates this information into changes in gene expression, are unexplored. Here, we demonstrate that, in the presence of $\mathrm{O}_{2}$, Apo-VqmA forms a C48-C63 intramolecular disulfide bond that restricts the ability of the protein to bind DNA. Formation of this bond is inhibited in the absence of oxygen or following supplementation of aerobic cells with a reductant, that, analogous to the absence of oxygen, generates a reducing environment. Thus, we propose that by interacting with the cell's redox environment, VqmA provides $V$. cholerae a mechanism to monitor oxygen levels (Figure 7A). In this context, we note that anaerobiosis causes a $\sim 7$-fold increase in Apo-VqmA-dependent pvqmR-lacZ reporter activity (Figure 3B). By contrast, DTT supplementation under aerobic conditions causes only an 3-fold increase in Apo-VqmA activity while the activity of Apo-VqmA C63A C134A, that lacks both disulfide bonds, is unchanged (Figure 5A). One interpretation of these data is that Apo-VqmA is responsive to additional oxygen-dependent stimuli that are not mimicked by DTT or by the inability to make disulfide bonds. We are currently testing this possibility.

With respect to DPO-bound VqmA we found that Holo-VqmA forms a C134-C134 inter-molecular disulfide bond that promotes its ability to activate transcription. 475 Formation of this bond is not modulated by oxygen levels, but is inhibited by the 476 reductant DTT. The absence of oxygen imposes a mildly reducing environment on cells, 477 while the presence of DTT imposes reductive stress $(42,58)$, suggesting that the C134478 C134 inter-molecular VqmA disulfide bond may allow V. cholerae to monitor reductive 479 stress. Collectively, we suggest a model in which cycling between multiple redox states, 480 namely Oxidized-Apo-VqmA, Reduced-Apo-VqmA, Oxidized-Holo-VqmA, and 481 Reduced-Holo-VqmA, enables V. cholerae to tune its QS-controlled collective behaviors 482 to a range of redox states (Figure 7B). There exist examples of individual disulfide 483 bonds restricting or enhancing the activity of transcription factors $(7,10,65)$. To our 
knowledge, however, this is the first example in which the same protein simultaneously uses two different disulfide bonds to modulate activity.

Bile is an abundant compound in the human small intestine that is well known to alter virulence in $V$. cholerae and other enteric pathogens such as Salmonella typhimurium and Shigella flexneri $(19,43)$. Bile is a heterogeneous mixture of molecules and studies have largely focused on defining the roles of individual components in bacterial physiology. Intriguingly, the individual components can drive opposing effects. In $V$. cholerae, bile fatty acids repress while the bile salt taurocholate induces virulence $(6,9,23,36,50,63)$. In our current study, we elected to use a mixture of bile salts reasoning that this strategy would more closely approximate what $V$. cholerae encounters in vivo. Our data suggest that bile salts disrupt the formation of the VqmA C134-C134 inter-molecular disulfide bond. We do not know the mechanism by which this occurs. However, previous studies show that bile salts, specifically cholic acid (CHO) and deoxycholic acid (DOC), interfere with redox homeostasis in Escherichia coli by shifting the cellular environment to an oxidizing one and fostering disulfide bond formation in cytosolic proteins (11). In the context of our work, since VqmA intermolecular disulfide bond formation is disrupted, we propose that application of a bile salts mixture to $V$. cholerae causes reductive stress. Consistent with this idea, taurocholate binds to and inhibits $\mathrm{DsbA}$, a protein required for the introduction of disulfide bonds in periplasmic proteins (62). We currently do not know whether incubation of $V$. cholerae with $\mathrm{CHO}$ and $\mathrm{DOC}$, rather than a bile salts mixture, would drive phenotypes mimicking those observed in E. coli.

What advantage does $V$. cholerae accrue by using the regulatory program uncovered in our study? We propose that $V$. cholerae uses the different blends of Als it encounters along with environmental modulation of VqmA activity to gauge its changing locations in the host. Thus, VqmA functions rather like a "GPS-device". In response to the information obtained about its micro-environment through VqmA, $V$. cholerae can appropriately tune its gene expression in space and time. We say this because, prior to entry into the small intestine (the site of cholera disease), $V$. cholerae will encounter oxygen limitation in the stomach. However, premature expression of virulence genes in the stomach, in the face of low $\mathrm{pH}$ and antimicrobial peptides would be unproductive 
515 and, moreover, divert energy from combating host defense systems. Thus, increased

516 VqmA activity, due to enhanced accumulation of DPO under anoxia coupled with

517 inhibition of formation of the activity-dampening intra-molecular disulfide bond, will

518 increase production of VqmR and, in turn, repress expression of genes involved in

519 biofilm formation and virulence. Anoxia and the concurrent presence of bile,

520 encountered upon entry into the host duodenum (upper region of the small intestine),

521 may provide a spatially relevant signal to alert $V$. cholerae to begin to express virulence

522 genes. In this case, bile-mediated inhibition of VqmA activity due to prevention of

523 formation of the inter-molecular disulfide bond will decrease VqmR production and, in

524 turn, enhance expression of genes involved in biofilm formation and virulence. The

525 combined use of bile and anoxia to decrease and increase VqmA activity, respectively,

526 is also noteworthy because, as $V$. cholerae proceeds further through the intestinal tract,

527 bile concentrations decrease near the ileum (lower portion of the small intestine), where

$528 \sim 95 \%$ of bile salts are reabsorbed, and they reach a minimum in the large intestine. By

529 contrast, anoxia is maintained throughout the intestinal tract. During successful

530 infection, $V$. cholerae cell numbers increase as disease progresses. Accumulation of

531 DPO should track with increasing cell density. Thus, it is possible that late in infection,

$532 V$. cholerae resides in a high DPO, anoxic environment lacking bile, conditions enabling

533 re-engagement of the VqmA-VqmR-DPO circuit, termination of virulence factor

534 production, and expulsion from the host. In this context, we also note that DPO

535 production by $V$. cholerae requires threonine as a substrate. Mucin is a major

536 constituent of the intestinal tract and is composed of $\sim 35 \%$ threonine (26). Intriguingly,

537 both the stomach and large intestine, locations where $V$. cholerae typically does not

538 reside, contain more mucus secreting glands and mucus layers than does the small

539 intestine (26). Thus, it is likely that enhanced access to mucus-derived threonine in the

540 large intestine allows $V$. cholerae to increase DPO production. Again, high DPO levels

541 repress virulence and biofilm formation. Thus, increased DPO production in the large

542 intestine would foster $V$. cholerae departure from the host. We speculate that, in

543 addition to oxygen and bile, the presence of mucus/threonine/the ability to synthesize

544 DPO is also leveraged by $V$. cholerae as an additional spatial cue to optimize host 545 dispersal timing. 
Materials and Methods:

Materials: iProof DNA polymerase was purchased from Biorad. Gel purification, plasmid-preparation, RNA-preparation (RNeasy), RNA-Protect reagents, qRT-PCR kits, and deoxynucleoside triphosphates were purchased from Qiagen. Antibodies were purchased from Sigma. Chitin flakes were supplied by Alfa Aesar. Instant Ocean (IO) Sea Salts came from from Tetra Fish. Bile salts were purchased from Fluka.

Bacterial growth: E. coli Top10 was used for cloning, E. coli S17-1 $\lambda$ pir was used for conjugations, and E. coli BL21 (DE3) was used for protein purification. V. cholerae and E. coli strains were grown in LB medium or in M9 minimal medium with glucose at $37^{\circ} \mathrm{C}$, with shaking. When required, media were supplemented with streptomycin, $500 \mu \mathrm{g} / \mathrm{mL}$; kanamycin $50 \mu \mathrm{g} / \mathrm{mL}$; spectinomycin, $200 \mu \mathrm{g} / \mathrm{mL}$, polymyxin B, $50 \mu \mathrm{g} / \mathrm{mL}$; and chloramphenicol, $1 \mu \mathrm{g} / \mathrm{mL}$. For bioluminescence assays, $V$. cholerae strains were cultured in SOC medium supplemented with tetracycline, and for Al-2 measurements, with boric acid $(20 \mu \mathrm{M})$. Unless otherwise indicated, bile salts were supplemented at $0.5 \% \mathrm{v} / \mathrm{v}$ and DPO at $25 \mu \mathrm{M}$. Where indicated, oxygen deprivation was achieved as follows: a) medium was sparged prior to use for at least 20 min with nitrogen gas; b) medium was incubated overnight with constant stirring inside a COY anaerobic chamber equipped with a catalyst to scavenge oxygen. For both (a) and (b), subsequent steps were conducted inside the COY anaerobic chamber and c) exponentially growing cells were transferred into capped microcentrifuge tubes with a headspace to volume ratio (HV ratio) of zero and anaerobiosis was verified by the addition of $0.001 \%$ resazurin to control tubes, as described previously $(18,37)$. Micro-aerobiosis was achieved at 10 min post-shift and anaerobiosis by $\sim 15-18$ min post-shift under these

572 conditions, consistent with previous observations (18). Experiments presented in Figure

5731 were conducted using strategy (a) and (b). Experiments in later sections used strategy

574 (c). In control experiments, we verified that our data were not altered due to differences 575 in growth in the presence or absence of oxygen (see Supplemental Figure 1B). 


\section{Strain construction:}

578 Chromosomal alterations: V. cholerae strains were constructed using natural579 transformation mediated multiplexed-genome-editing (MuGENT) (12, 13). Unless 580 otherwise stated, chromosomal DNA from $V$. cholerae $66706 \mathrm{Sm}^{R}$ was used as a 581 template for PCR reactions. DNA fragments containing $\sim 3 \mathrm{~kb}$ of homology to the 582 upstream and downstream regions of the desired chromosomal region were generated 583 using PCR. When necessary, SOE PCR was used to combine multiple fragments of 584 DNA, in which each fragment typically contained 27-30 bp of overhang homology (12, 585 13). Antibiotic resistance cassettes, to facilitate selection of transformants following the 586 MuGENT step, were designed to integrate at a neutral locus (vc1807) and were gifts 587 from the Dalia group (Indiana University) $(12,13)$. V. cholerae cultures for use in natural 588 transformations were prepared by inoculating $1 \mathrm{~mL}$ liquid LB medium from freezer stocks and growing the cells to $\mathrm{OD}_{600} \sim 1$. Cells were pelleted at maximum speed in a 590 micro-centrifuge and resuspended at the original volume in $1 \mathrm{X} 1 \mathrm{O}$ Sea Salts $(7 \mathrm{~g} / \mathrm{L})$. 591 Competence was induced by combining a $75 \mu \mathrm{L}$ aliquot of the cell suspension with 900 $592 \mu \mathrm{L}$ of a chitin 10 mixture (8 $\mathrm{g} / \mathrm{L}$ chitin), and the preparation was incubated overnight at $59330^{\circ} \mathrm{C}$. The next day, these mixtures were supplemented with one (or multiple) PCR594 amplified linear DNA fragment(s) of interest, as well as DNA encoding an antibiotic resistance cassette $(12,13)$. These mixtures were incubated overnight at $30^{\circ} \mathrm{C}$, followed by vortex for $10 \mathrm{~min}$. Next, $150 \mu \mathrm{L}$ of the suspension was plated onto solid LB medium containing the appropriate antibiotics followed by overnight incubation at $30^{\circ} \mathrm{C}$.

598 Resulting transformants were passaged three times on solid LB medium with antibiotics 599 for purification. Genomic DNA from recombinant strains was used as a template for 600 PCR to generate DNA fragments for future co-transformation, when necessary.

601

602 Site-directed mutations in vqmA were constructed by incorporating the desired 603 alteration into forward or reverse PCR primers and generating DNA fragments with 604 homology to DNA flanking chromosomal vqmA. These fragments were transformed, as 605 described above, into a strain carrying vmqA::kan and the vqmR-lacZ transcriptional 606 reporter integrated onto the chromosome. Clones were selected by screening for loss of 
607 kanamycin resistance and/or by assessment of positive LacZ activity when plated on 608 agar containing $50 \mu \mathrm{g} / \mathrm{mL}$ X-gal.

609

610

611

612

613

614

615

616

617

618

619

620

621

622

623

624

625

626

627

628

629

630

631

632

633

634

635

636

637

Plasmid constructions: DNA cloned into the pBAD-pEVS or pEVS plasmids was assembled using enzyme-free XthA-dependent in vivo recombination cloning, as previously described $(3,41)$. Briefly, linear insert DNA fragments containing 30 base pairs of overlapping homology were generated using PCR. The plasmid backbone was likewise linearized by PCR-amplification. All DNA fragments were gel purified and eluted in $\mathrm{ddH}_{2} \mathrm{O}$. Thereafter, $80 \mathrm{ng}$ of the backbone and $240 \mathrm{ng}$ of each insert DNA fragment were combined, incubated for $1 \mathrm{~h}$ at room temperature followed by transformation and clone recovery in chemically-competent Top10 E. coli cells. Constructs in the pET15b backbone were assembled using traditional restrictionenzyme cloning using primers and protocols described earlier (46).

Assessing protein abundance and formation of disulfide bonds: Strains cultured overnight in LB medium ( 16-18 h) were diluted into fresh M9 minimal medium, with antibiotics, as necessary, to a final $O D_{600}=0.004$. When assessing levels of VqmA-FLAG produced from the chromosomally-integrated vqmA-FLAG construct, strains were cultured to $\mathrm{OD}_{600} \sim 0.3$ ( $\sim 4 \mathrm{~h}$ of growth) and cells were harvested by centrifugation. For strains requiring induction of protein expression, $0.2 \%$ arabinose was added to the culture medium at $4 \mathrm{~h}$ post-inoculation and growth was continued for an additional $1 \mathrm{~h}$ at which point the cultures were divided and portions were supplemented with $25 \mu \mathrm{M}$ DPO and/or $0.5 \%$ bile salts and/or deprived of oxygen. Treatments were continued for another $1 \mathrm{~h}$ after which cells were harvested by centrifugation at 13,000 rpm and the pellets were immediately frozen at $-80^{\circ} \mathrm{C}$ until use.

Immunoblotting: Cells were resuspended in ice-cold PBS and diluted to a final $\mathrm{OD}_{600}=7$ for protein produced from the chromosome or to $\mathrm{OD}_{600=3.5}$ for protein produced from a plasmid, in a volume of $20 \mu \mathrm{L}$. The cells were lysed by addition of $5 \mu \mathrm{L}$ Bug Buster (Novagen) supplemented with $1 \mu \mathrm{g}$ lysozyme, and $25 \mathrm{U} / \mathrm{mL}$ benzonase. Samples were combined with SDS-PAGE buffer in the presence or absence of DTT 
638

639

640

641

642

643

644

645

646

647

648

649

650

651

652

653

654

655

656

657

658

659

660

661

662

663

664

665

666

667

668

(100 mM), boiled for $20 \mathrm{~min}$, and proteins separated on 4-20\% Mini-Protein TGX gels (Biorad). Proteins were transferred to PVDF membranes (Bio-Rad) for $1 \mathrm{~h}$ at $4^{\circ} \mathrm{C}$ at 100 V. Membranes were blocked overnight in PBST (1X PBS, 0.03\% Tween-20) supplemented with $5 \%$ milk, washed 5 times with PBST, and incubated for 40 min with 1:5000 dilution of monoclonal Anti-FLAG-Peroxidase antibody (Sigma) in PBST. The membranes were subsequently washed another five times with PBST. FLAG epitopetagged protein levels were visualized using the Amersham ECL western blotting detection reagent (GE Healthcare). Thereafter, the antibody was removed by 2 serial incubations in stripping buffer (15 g/L glycine, $1 \mathrm{~g} / \mathrm{L}$ SDS, $10 \mathrm{~mL} / \mathrm{L}$ Tween-20, pH 2.2) for 5 min each. The membrane was re-equilibrated by 4 washes in PBST, 20 min each, and used to detect the abundance of the loading control, RNA Polymerase $\alpha$. Washes and incubations as described above were performed to enable antibody binding and removal of excess. The primary antibody, anti-E. coli RNA Polymerase a (Biolegend), and the secondary antibody, anti-mouse IgG HRP conjugate antibody (Promega), were both used at a 1:10,000 dilutions. In all cases, protein levels were quantified using Image $\mathrm{J}$ software.

Protein purification: pET15b plasmids encoding $6 \mathrm{XHis-VqmA}$ were mobilized into $\Delta t d h$ E. coli BL21 DE3. Strains were cultured for protein production as described previously $(22,46)$. Cells were harvested by centrifugation and pellets were resuspended in 1/100 volume of lysis buffer (50 mM Tris, $150 \mathrm{mM} \mathrm{NaCl}, \mathrm{pH} 7.5$ containing $0.5 \mathrm{mg} / \mathrm{mL}$ lysozyme, $1 \mathrm{X}$ protease inhibitor, and benzonase) for five min followed by the addition of an equal volume of Bugbuster Reagent (Novagen). The cell lysate was clarified by centrifugation at $13,000 \mathrm{rpm}$ and protein was purified using $\mathrm{Ni}$ NTA superflow resin (Qiagen), according to the manufacturer's recommendations for a centrifugation-based protocol, except that the loading and wash buffers all contained 1 $5 \mathrm{mM}$ imidazole to decrease non-specific protein binding. The protein was eluted from the resin using $300 \mathrm{mM}$ imidazole and thereafter dialyzed twice against $50 \mathrm{mM}$ Tris, 150 $\mathrm{mM} \mathrm{NaCl}, \mathrm{pH}$ 7.5, using a Slide-A-Lyzer module (Thermo Fisher). When necessary, buffers were amended with $5 \mathrm{mM}$ DTT. To purify Holo-VqmA, buffers were supplemented with $100 \mu \mathrm{M}$ DPO. 
670 Electromobility gel shift assays (EMSA): The DNA corresponding to the promoter 671 region of $v q m R, \sim 100$ base pairs, was amplified using $V$. cholerae genomic DNA as a 672 template. Where mentioned, protein was pre-treated in binding buffer with 10-fold molar excess of DTT or diamide in order to reduce or oxidize the protein, respectively. To 674 initiate EMSA assays, 0.2-3.5 $\mu \mathrm{M}$ protein was combined with $30 \mathrm{ng}$ probe DNA in 675 binding buffer $(50 \mathrm{mM}$ Tris- $\mathrm{HCl} \mathrm{pH} \mathrm{8,} 150 \mathrm{mM} \mathrm{NaCl})$. Reactions were allowed to 676 proceed at RT for 15 min. Samples were separated on a Novex 6\% DNA Retardation 677 Gel (Thermo) by electrophoresis in 1X TBE at 100 V. Gels were subsequently incubated 678 with Sybr Green reagent, diluted in 1X TBE at RT for $25 \mathrm{~min}$, washed with five 679 successive rounds of $\mathrm{ddH}_{2} \mathrm{O}$, and imaged using an ImageQuant LAS 4000 imager and the Sybr Green channel setting.

Analysis of relative Al levels in conditioned growth medium following aerobic or anaerobic growth: $V$. cholerae strains were cultured overnight in LB medium ( 16-18 h), diluted into fresh aerobic or anaerobic LB medium to a final $O_{600}=0.004$. The cultures were incubated at $37^{\circ} \mathrm{C}$ for an additional $\sim 6 \mathrm{~h}$ with shaking. The cells were removed by centrifugation at 13,000 rpm and the spent medium filtered through $0.2 \mu \mathrm{m}$ filters. 20\% (v/v) 5X LB was added to the spent medium preparations (hereafter designated as reconditioned spent medium). Negative controls consisted of spent medium prepared from strains incapable of synthesizing CAl-1, Al-2, and DPO. Subsequently, reconditioned spent medium was combined with $V$. cholerae reporter strains expressing only a single QS receptor that, therefore detect only one AI. In the case of CAl-1 and Al-2 detection, the reporter strains carried a plasmid encoding the $V$. harvyei luxCDABE (luciferase) operon (39). For DPO detection, the reporter strain possessed a pvqmR-lux (luciferase) fusion on the chromosome in place of the native 695 lacZ locus (22). Genotypes are provided in Table S1. The reporter strains and reconditioned spent medium preparations were combined to a final volume of $150 \mu \mathrm{L}$ in wells of 96-well plates, covered with Breathe-Easy Film, and incubated at $30^{\circ} \mathrm{C}$ with shaking for 2.5-4 h. Finally, bioluminescence and $\mathrm{OD}_{600}$ values were recorded. Relative 
700

701

702

703

704

705

706

707

708

709

710

711

712

713

714

715

716

717

718

719

720

721

722

723

724

725

726

727

728

729

730

Normalized RLUs were obtained by subtraction of the RLU values obtained from the negative controls.

Beta-galactosidase assays: $V$. cholerae strains cultured overnight in LB medium were diluted into fresh $\mathrm{M} 9$ medium to a final $\mathrm{OD}_{600}=0.004$ and thereafter cultured to $\mathrm{OD}_{600} \sim 0.3(\sim 4 \mathrm{~h})$. The cultures were held on ice prior to assay or subjected to further treatments, as described below. For strains requiring inducible protein expression, $0.01 \%$ arabinose was added to the culture medium at $4 \mathrm{~h}$ post-inoculation and growth was continued for an additional $1 \mathrm{~h}$. In all cases, cultures were divided into aliquots and individual portions were supplemented with $25 \mu \mathrm{M}$ DPO and/or $0.5 \%$ bile salts and/or deprived of oxygen. Cells were cultured for an additional $1 \mathrm{~h}$ and then held on ice prior to assay. To assess the effect of reductant, at $2 \mathrm{~h}$ post-inoculation, the cultures were supplemented with $300 \mu \mathrm{M}$ DTT. Cells were cultured for another $2 \mathrm{~h}$ to allow DTT permeation, before vqmA expression was induced by the addition of arabinose. Subsequent treatments were as above. LacZ activity assays were carried out as follows: cells were combined 1:1 (v/v) with Bugbuster Reagent for $20 \mathrm{~min}$. The assay was initiated by combining $20 \mu \mathrm{L}$ of the cell/Bugbuster mixture with $140 \mu \mathrm{L}$ of assay buffer (80\% Bugbuster, 10\% 10X PBS, 1 mM MgSO $4,10 \mu \mathrm{g} / \mathrm{mL}$ lysozyme, benzonase $(0.05 \% \quad \mathrm{v} / \mathrm{v}), \quad \beta$-mercaptoethanol $(0.1 \% \quad \mathrm{v} / \mathrm{v}), 67 \mu \mathrm{g} / \mathrm{mL}$ fluorescein-di- $\beta$-Dgalactopyranoside). Changes in fluorescence were captured using the GFP channel on a Synergy Neo2 HTS Multi-Mode Microplate Reader. Activity units were defined as the change in fluorescence/minute/ $O D_{600}$ of the culture at the point of harvest.

RNA isolation and quantitative RT-PCR. Strains cultured overnight in LB were diluted into fresh $M 9$ minimal medium to a final $O D_{600}=0.004$. Next, the strains were grown to $\mathrm{OD}_{600} \sim 0.3$ ( $\sim \mathrm{h}$ post-inoculation) with shaking at $37^{\circ} \mathrm{C}$ at which point the cultures were divided into portions that were supplemented with $25 \mu \mathrm{M}$ DPO and/or $0.5 \%$ bile salts and/or deprived of oxygen. Treatments were continued for another $1 \mathrm{~h}$, the cells were harvested and treated for 15 min at room temperature with RNAProtect reagent, as per the manufacturer's instructions. RNA was isolated using the RNeasy kit (Qiagen) and 2 $\mu \mathrm{g}$ of total RNA was depleted of contaminating DNA using TurboDNase (Applied 
731 Biosystems), using the manufacturer's recommended protocol. $500 \mathrm{ng}$ of the resulting

732 total RNA was used to construct cDNA libraries using SuperScript III Reverse

733 Transcriptase (Invitrogen). q-PCR was conducted using the PerfeCTa SYBR Green

734 FastMix Low ROX (Quanta Biociences) reagent.

735

736

Caco-2 culture and co-culture with bile and $\boldsymbol{V}$. cholerae. The HTB37 cell line was obtained from ATCC and thereafter cultured and passaged in EMEM (ATCC) supplemented with an 10\% FBS (Thermo Fisher), 2 mM glutamine (Thermo Fisher), 1X Penstrep (Thermo Fisher) and $2.5 \mu \mathrm{g} / \mathrm{mL}$ plasmomycin (Invitrogen), as per ATTC recommendations. Prior to co-culture, Caco-2 cells were seeded at $0.32 \mathrm{~cm}^{2}$ into a tissue culture treated 96-well plate in EMEM medium, as above, except that the $2 \mathrm{mM}$ glutamine was not included. The cells were cultured to confluency, the medium was removed by aspiration, and the cells were washed with Earle's balanced salt solution followed by the addition of EMEM medium containing $25 \mu \mathrm{M}$ DPO, but lacking antibiotics/glutamine/FBS. At time zero, V. cholerae, grown as described below, was added, at an $\mathrm{MOI}$ of 10 and with $0.05 \%$ bile salts. Following co-culture for $3.5 \mathrm{~h}$, the medium and the bacteria were removed by aspiration, the wells were washed with Earle's balanced salt solution, and Caco-2 cell viability was assessed using the neutral red assay (51). For co-culture with Caco-2 cells, V. cholerae WT and $\triangle v q m A$ strains were cultured overnight under static conditions in AKI medium containing $25 \mu \mathrm{M}$ DPO in tubes with an $\mathrm{HV}$ ratio of zero at $37^{\circ} \mathrm{C}$. The next day, the cells were decanted into glass tubes and cultured with vigrous shaking for $1 \mathrm{~h}$. Subsequently, the cultures were diluted with PBS to the appropriate density and added to the Caco-2 cells.

Acknowledgements: We thank the Donia lab for generously allowing us to use their anaerobic chamber. We thank Dr. Ankur Dalia for the gift of protocols, strains, and reagents to facilitate MuGENT cloning. We thank Dr. Tharan Srikumar, Saw Kyin, and Henry Shwe for help with mass spectroscopy experiments. This work was supported by

759 the Howard Hughes Medical Institute, National Science foundation Grant MCB760 1713731, and NIH Grant 5R37GM065859 to B.L.B. A.A.M is a Howard Hughes Medical Institute Fellow of the Life Sciences Research Institute. 
bioRxiv preprint doi: https://doi.org/10.1101/2020.05.04.076810; this version posted May 4, 2020. The copyright holder for this preprint (which was not certified by peer review) is the author/funder, who has granted bioRxiv a license to display the preprint in perpetuity. It is made available under aCC-BY-NC-ND 4.0 International license.

762

763 Competing interests: The authors have no competing interests to declare.

764 


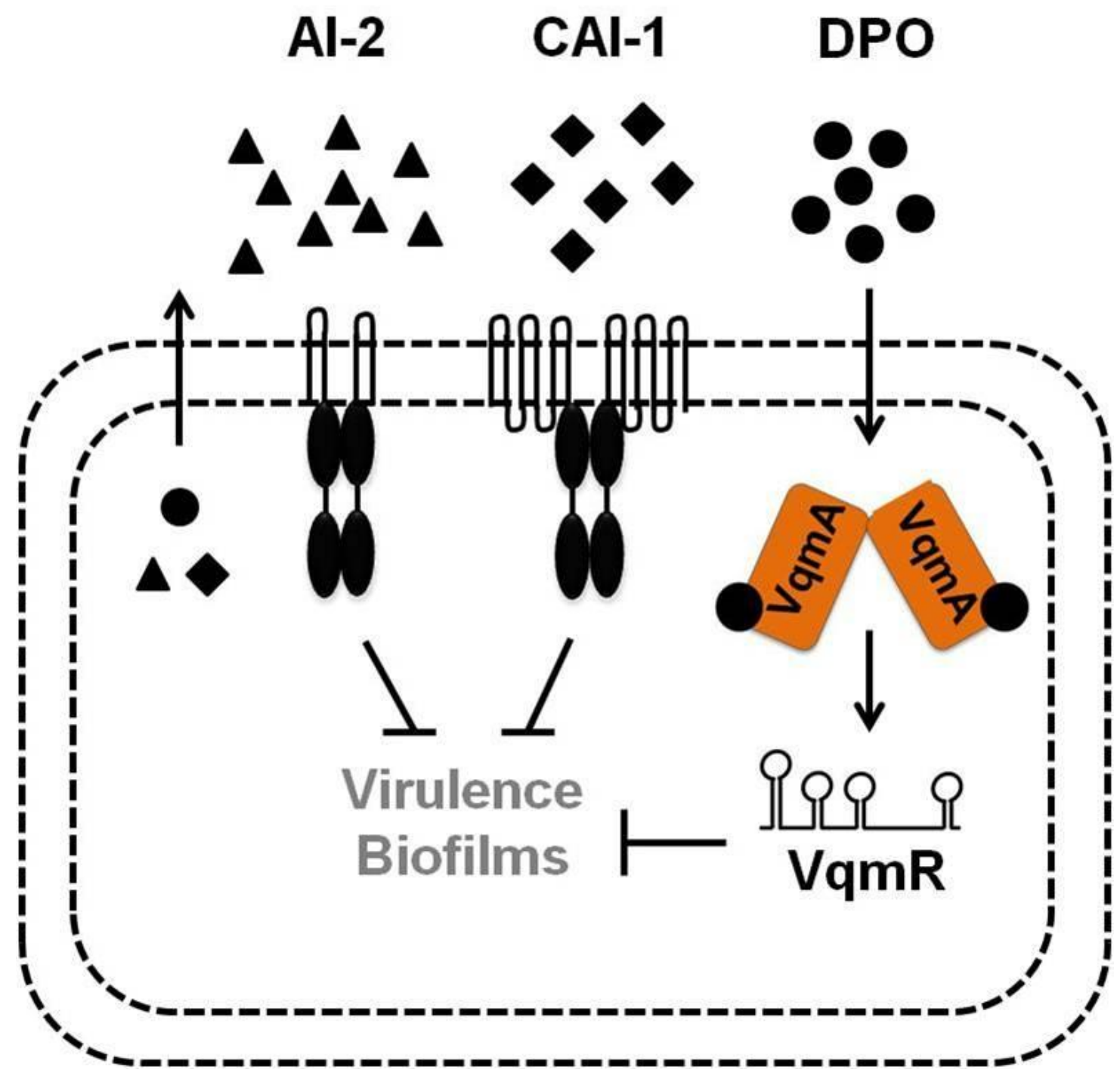

Figure 1. Simplified V. cholerae quorum-sensing pathways. See text for details. 

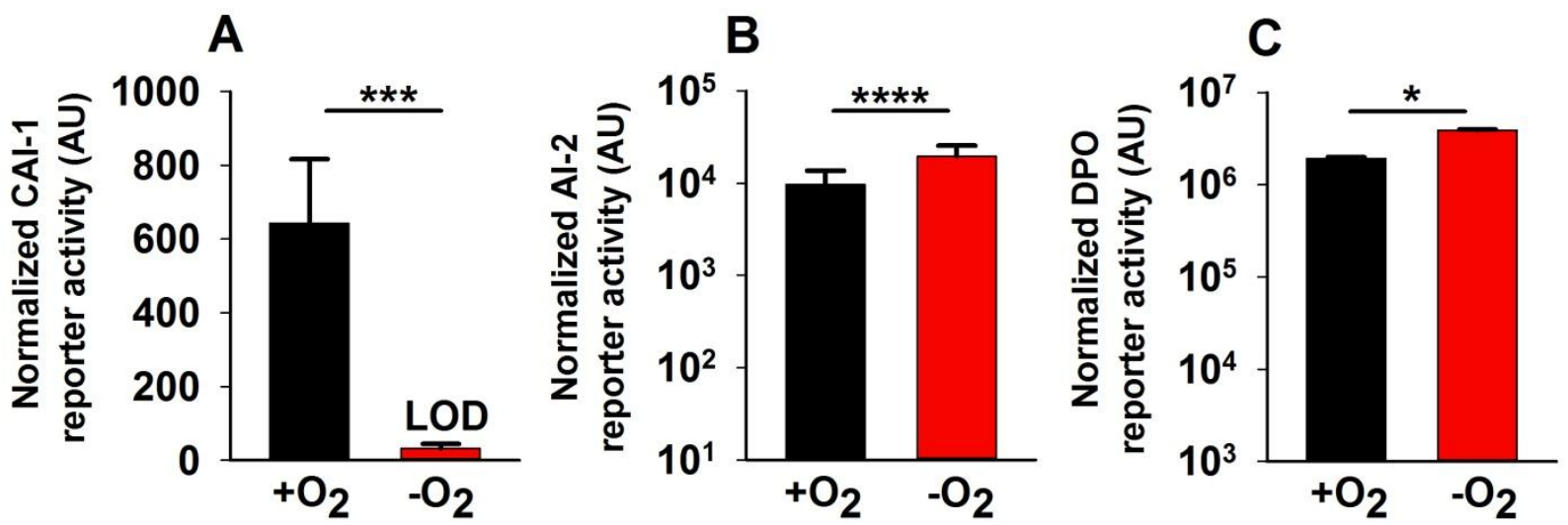

770 Figure 2. Oxygen deprivation modulates V. cholerae Al production. (A) $80 \%$, (B) 25\%, 771 or (C) $30 \%$ cell-free culture fluids prepared from WT V. cholerae grown in the presence 772 or absence of $\mathrm{O}_{2}$ were provided to $\mathrm{V}$. cholerae reporter strains that produce 773 bioluminescence in response to exogenous (A) CAl-1, (B) Al-2, or (C) DPO. Data 774 represent the average values of biological replicates $(n=3)$ and error bars represent SD. $775 *$ denotes $p<0.05$, ** denotes $p<0.001$, and ${ }^{* * * *}$ denotes $p<0.0001$. 


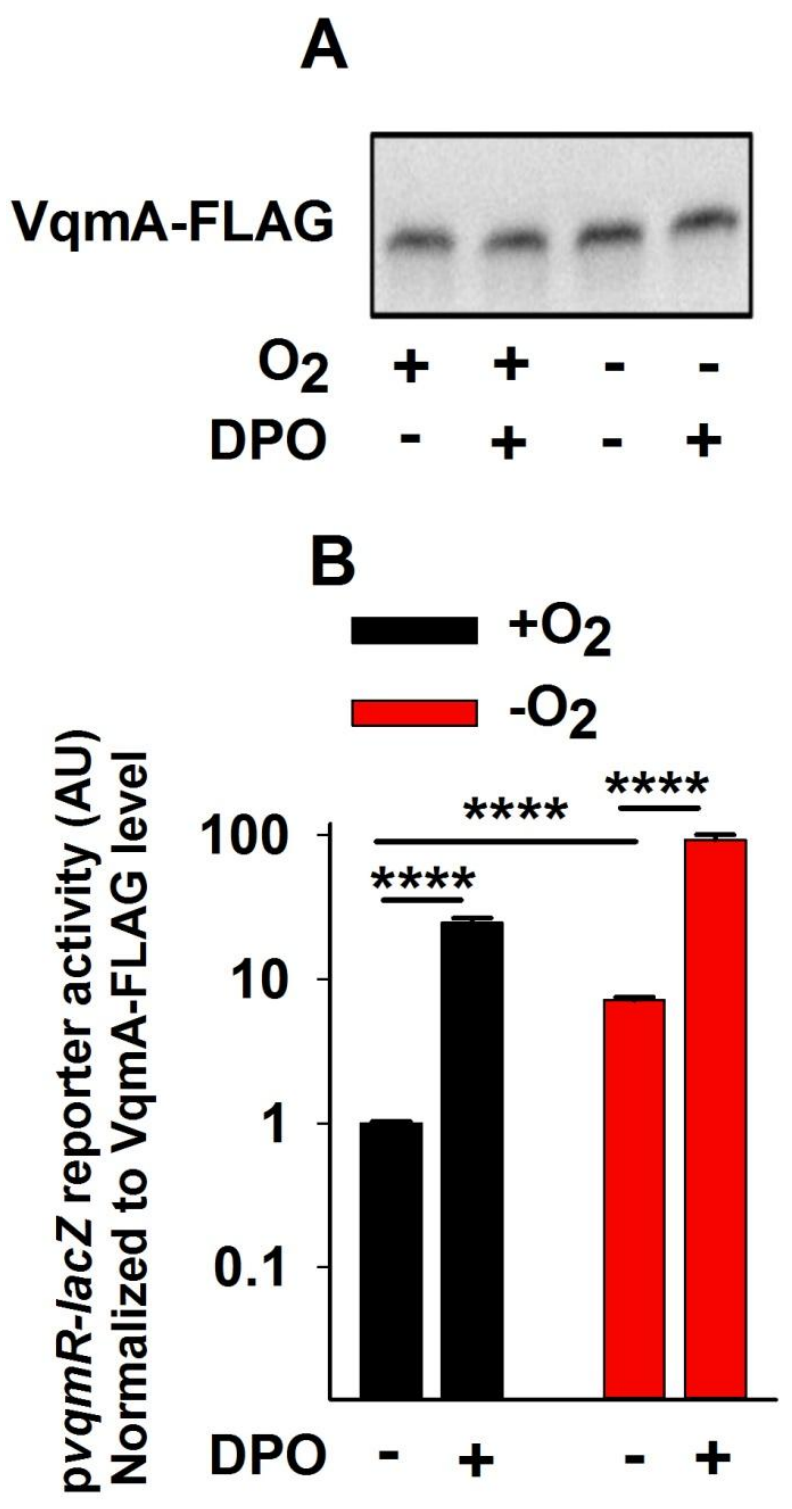

779 Figure 3. Apo- and Holo-VqmA activities are enhanced in the absence of oxygen. (A) 780 Western blot showing VqmA-FLAG abundance in the $\Delta v q m A \Delta t d h$. cholerae carrying 781 pvqmA-FLAG and treated as shown for $1 \mathrm{~h}$. DPO was supplied at $25 \mu \mathrm{M}$ DPO. (B) 782 Transcriptional activity for the pvqmR-lacZ reporter from the same samples presented in 783 A. Data in B were normalized to the level of VqmA-FLAG in A. In B, data represent the 784 average values of biological replicates $(n=3)$ and error bars represent SD. ${ }^{* * * *}$ denotes $785 \mathrm{p}<0.0001$. 

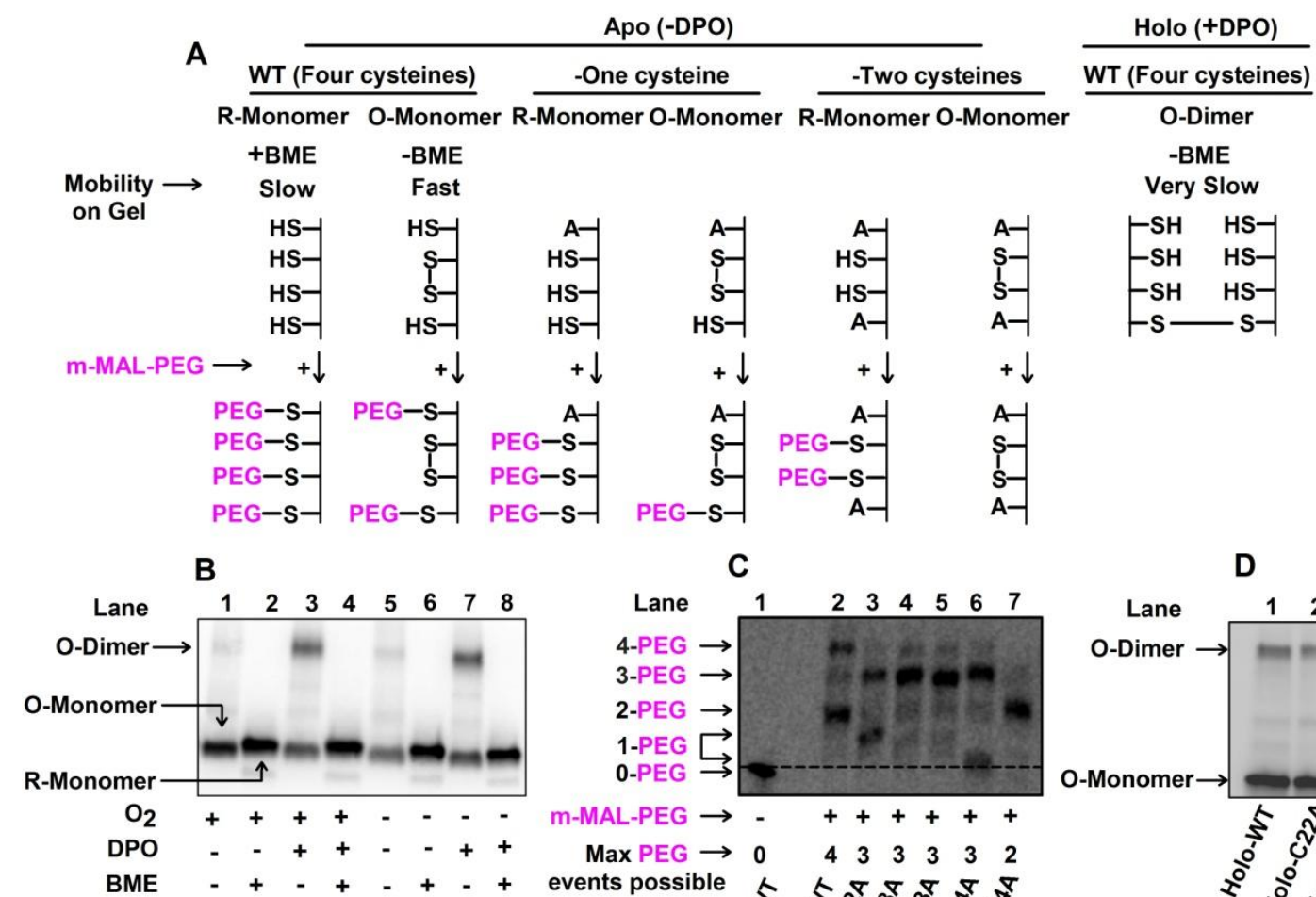

C
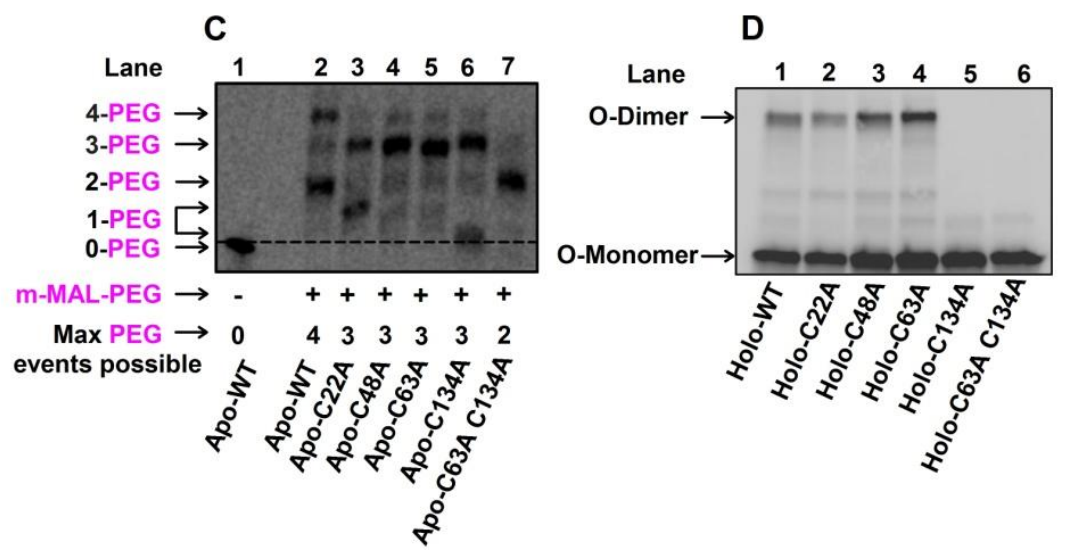

Figure 4. VqmA forms intra- and inter-molecular disulfide bonds in an oxygen- and DPO-dependent manner. (A) Schematic depicting the oxidized and reduced forms of VqmA (top) and the strategy employed to interrogate intra-molecular disulfide bond formation by trapping reduced thiols with methoxypolyethylene glycol maleimide ( $\mathrm{m}$ MAL-PEG; 5kDa, bottom) (32). (B) Western blot showing VqmA-FLAG protein produced by the $\triangle v q m A \Delta t d h V$. cholerae strain carrying pvqmA-FLAG following the specified treatments. (C) Western blot showing VqmA-FLAG protein produced by the $\triangle$ vqmA $\Delta t d h V$. cholerae strain carrying the designated pvqmA-FLAG alleles following treatment with m-MAL-PEG (32) in the absence of DPO. Note that in lanes 3 and 6 , the proteins containing 1-PEG modification migrate somewhat differently. We infer the bands to be 797 1-PEG events by comparison with the 0-PEG event in lane 1 and the 2-PEG event in 798 lane 7. A likely explanation is that the protein containing 1-PEG in lane 6 adopts a more compact conformation than the modified protein in lane 3 and so it migrates faster through the gel $(2,55)$. The dotted line distinguishes the 0-PEG VqmA from the lowest 
bioRxiv preprint doi: https://doi.org/10.1101/2020.05.04.076810; this version posted May 4, 2020. The copyright holder for this preprint (which was not certified by peer review) is the author/funder, who has granted bioRxiv a license to display the preprint in perpetuity. It is made available under aCC-BY-NC-ND 4.0 International license.

801 1-PEG decorated species. (D) Western blot showing VqmA-FLAG for the strains in C 802 following supplementation with $25 \mu \mathrm{M}$ DPO under non-reducing conditions. 


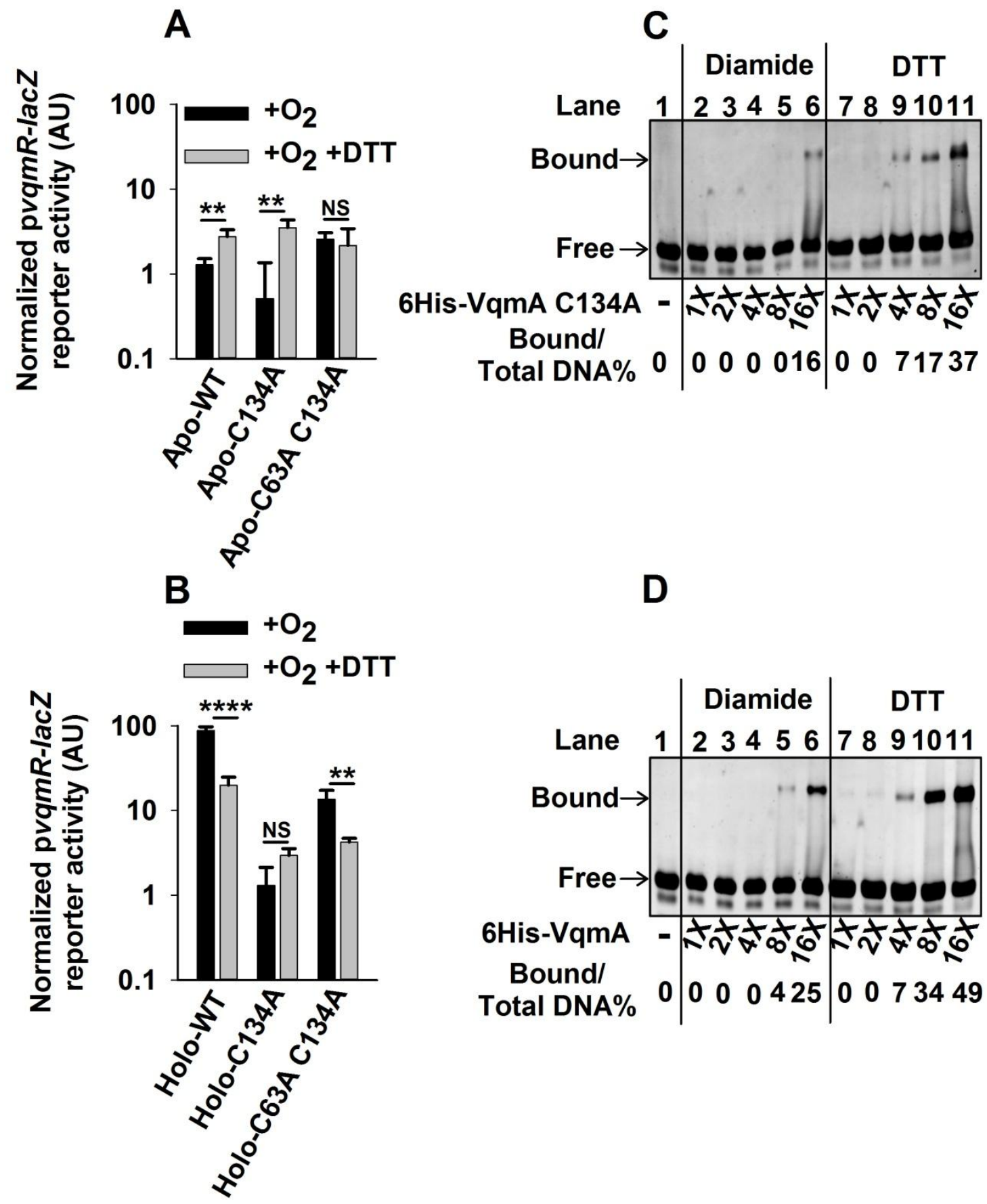

821 Figure 5. VqmA activity is differentially modulated by the cellular redox environment 822 and intra- and inter-molecular disulfide bonds. (A) pvqmR-lacZ activity in $\Delta v q m A \Delta t d h$ 823 V. cholerae carrying the designated pvqmA-FLAG constructs following growth in the 824 presence of $\mathrm{O}_{2}$ without (black) or with DTT (gray). (B) Strains cultured as in $A$ 
825 supplemented with $25 \mu \mathrm{M}$ DPO. (C) Electromobility shift analysis (EMSA) of 6 His-VqmA 826 C134A binding to vqmR promoter DNA. (D) EMSA as in C for WT 6His-VqmA. In C and 827 D, all lanes contained $35 \mathrm{ng}$ of promoter DNA and, where indicated, dilutions of protein 828 were used with $16 \mathrm{X}=2 \mu \mathrm{g}$. Bound and free correspond to DNA that is and is not bound 829 to VqmA protein, respectively. As indicated, VqmA had been treated with 10-fold molar 830 excess of diamide or DTT. Data in A and B represent average values of biological 831 replicates $(n=3)$ and error bars represent SD. ${ }^{* *}$ denotes $p<0.01,{ }^{* * *}$ denotes $p<0.0001$, 832 and NS denotes $\mathrm{p}>0.05$. 

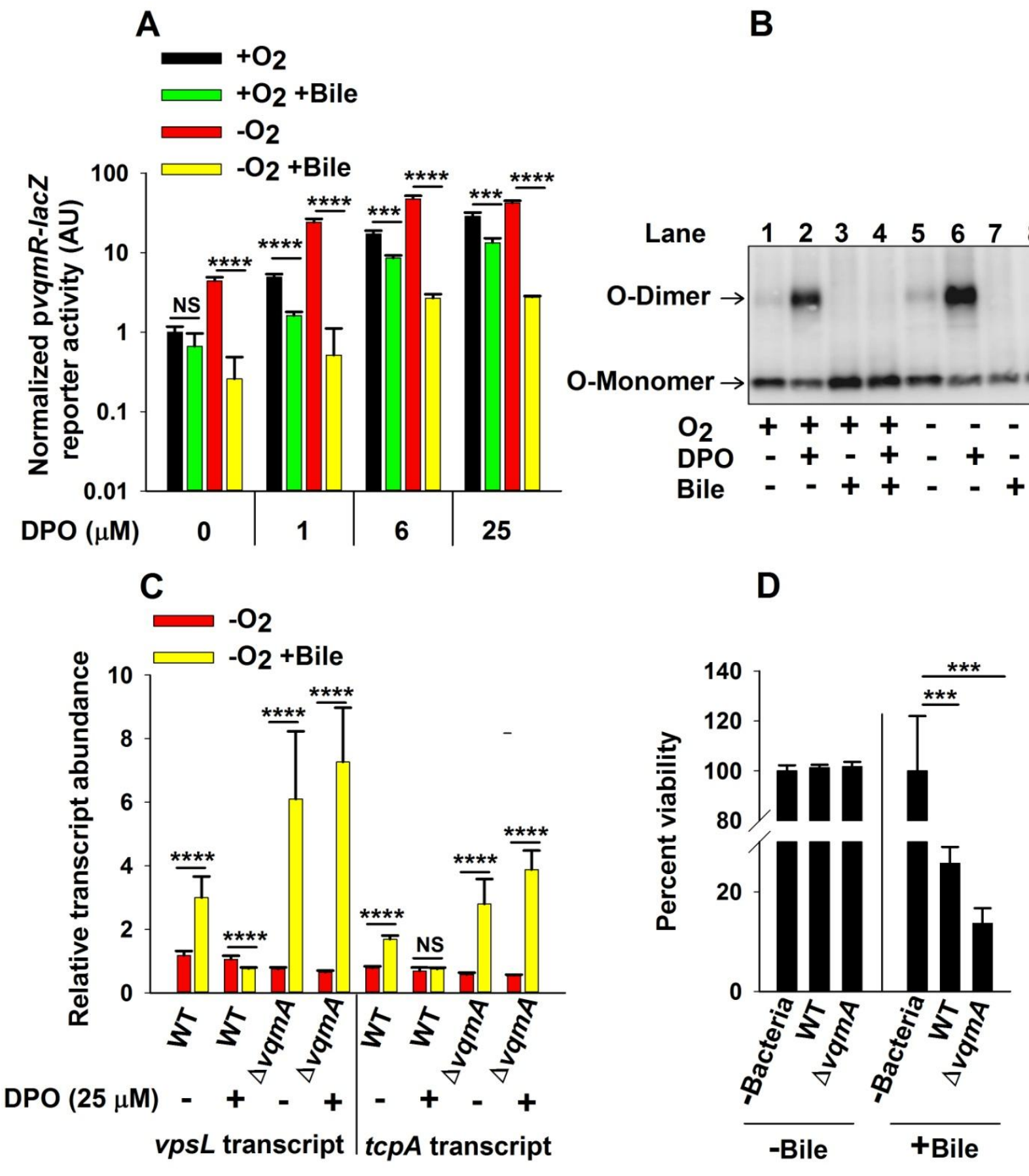

835

Figure 6. Bile salts disrupt VqmA-DPO-mediated signal transduction and promote $V$. cholerae virulence. (A) pvqmR-lacZ activity in $\Delta v q m A \Delta t d h$. cholerae carrying pvqmA-

837 FLAG following $1 \mathrm{~h}$ in the presence or absence of $\mathrm{O}_{2}, 0.5 \%(\mathrm{v} / \mathrm{v})$ bile, and 1-25 $\mu \mathrm{M}$ DPO 838 or combinations of all three treatments as indicated. (B) Western blot showing VqmA839 FLAG for the strain in A following the indicated treatments. (C) Relative expression 840 levels of $v p s L$ and $t c p A$ in WT and $\triangle v q m A V$. cholerae following the designated 
841 treatments. (D) Viability of human intestinal CaCo-2 cells in the absence of bacteria or

842 following challenge by WT or $\triangle$ vqmA $V$. cholerae and in the presence or absence of $8430.05 \%$ bile. Data in panels $A$, and $D$ represent average values of biological replicates $844(n=3)$ and error bars represent SD. Data in panel $C$ represent the average value of three 845 biological replicates and two technical replicates for each sample $(n=6)$ and error bars 846 represent SD. ${ }^{* * *}$ denotes $p<0.001,{ }^{* * * *}$ denotes $p<0.0001$, and NS denotes $p>0.05$. 


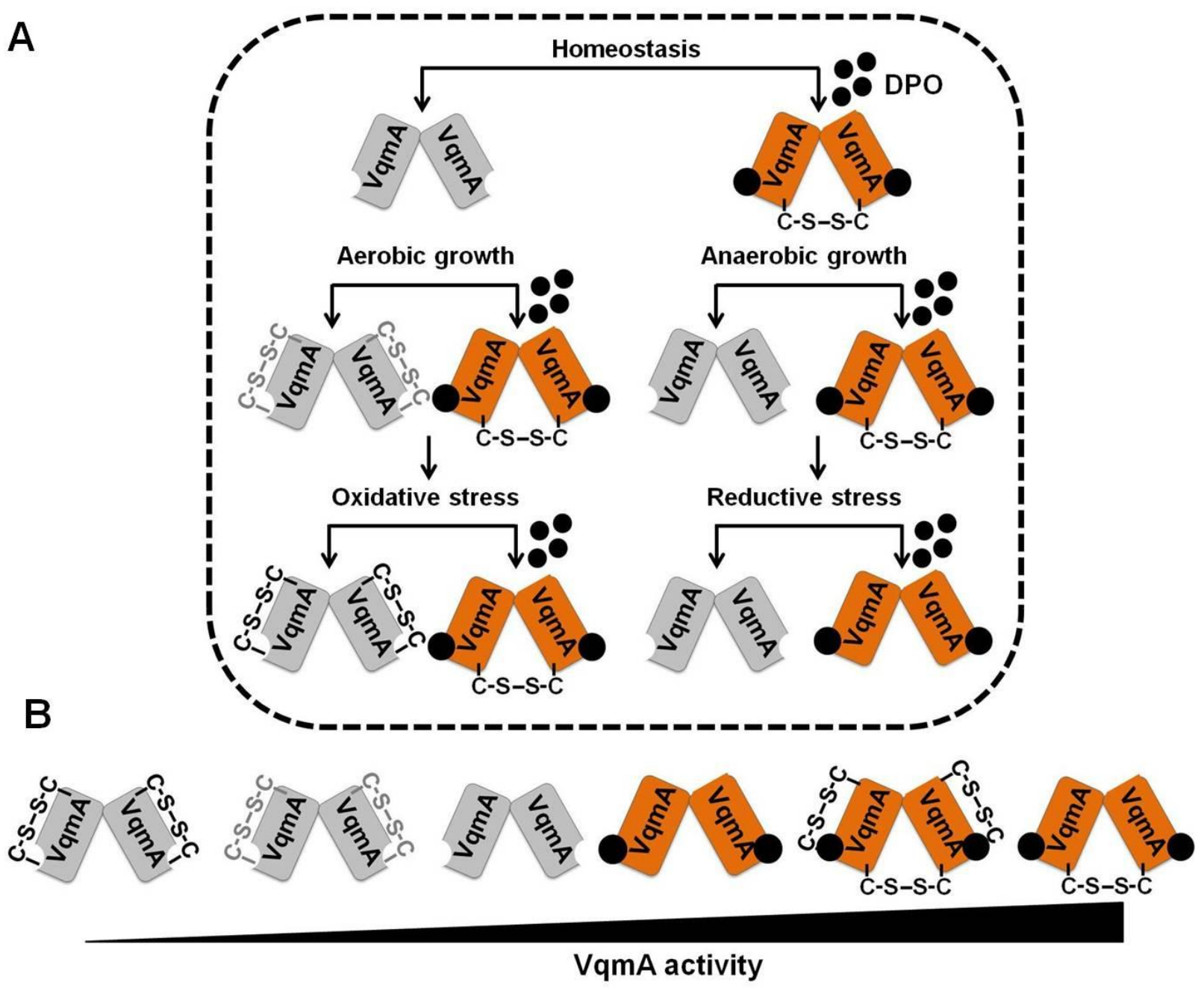

849 Figure 7. Model depicting VqmA as a hub protein that compiles quorum-sensing, 850 environmental, and host information. (A) VqmA can exist in different states in vivo 851 depending upon the availability of the DPO ligand and the cellular redox state. Thus, 852 Apo-VqmA forms the C48-C63 intra-molecular disulfide bond that suppresses its ability 853 to bind DNA. Apo-VqmA activity is high under reducing growth conditions in which 854 formation of the intra-molecular C48-C63 bond is inhibited. Holo-VqmA forms the C134855 C134 inter-molecular disulfide bond that promotes DNA binding. Reductive stress 856 disrupts the formation of the inter-molecular disulfide bond. We propose that growth in 857 the presence of bile salts imposes reductive stress, disrupts the formation of the C134858 C134 inter-molecular disulfide bond, and restricts VqmA DNA binding, thereby 859 promoting virulence and biofilm formation. The gray and black intra-molecular disulfide 860 bonds denote partially-oxidized and fully-oxidized VqmA, respectfully. (B) Relative 
bioRxiv preprint doi: https://doi.org/10.1101/2020.05.04.076810; this version posted May 4, 2020. The copyright holder for this preprint (which

was not certified by peer review) is the author/funder, who has granted bioRxiv a license to display the preprint in perpetuity. It is made available under aCC-BY-NC-ND 4.0 International license.

861 VqmA activity levels as a consequence of disulfide bond formation. For simplicity, the 862 fourth and fifth species in B are not displayed in A.

863 


\section{References:}

865

866

867

868

869

870

871

872

873

874

875

876

877

878

879

880

881

882

883

884

885

886

887

888

889

890

891

892

893

894

895

896

897

898

899

900

901

902

903

904

905

906

1. Antelmann, H., and J. D. Helmann. 2010. Thiol-based redox switches and gene regulation. Antioxid Redox Signal 14:1049-63.

2. Bardwell, J. C., K. McGovern, and J. Beckwith. 1991. Identification of a protein required for disulfide bond formation in vivo. Cell 67:581-9.

3. Beyer, H. M., P. Gonschorek, S. L. Samodelov, M. Meier, W. Weber, and M. D. Zurbriggen. 2015. AQUA Cloning: A Versatile and Simple Enzyme-Free Cloning Approach. PLoS One 10:e0137652.

4. Braun, M., and L. Thony-Meyer. 2005. Cytochrome c maturation and the physiological role of c-type cytochromes in Vibrio cholerae. J Bacteriol 187:59966004.

5. Bridges, A. A., and B. L. Bassler. 2019. The intragenus and interspecies quorum-sensing autoinducers exert distinct control over Vibrio cholerae biofilm formation and dispersal. PLoS Biol 17:e3000429.

6. Chatterjee, A., P. K. Dutta, and R. Chowdhury. 2007. Effect of fatty acids and cholesterol present in bile on expression of virulence factors and motility of Vibrio cholerae. Infect Immun 75:1946-53.

7. Chen, P. R., S. Nishida, C. B. Poor, A. Cheng, T. Bae, L. Kuechenmeister, P. M. Dunman, D. Missiakas, and C. He. 2009. A new oxidative sensing and regulation pathway mediated by the MgrA homologue SarZ in Staphylococcus aureus. Mol Microbiol 71:198-211.

8. Chen, X., S. Schauder, N. Potier, A. Van Dorsselaer, I. Pelczer, B. L. Bassler, and F. M. Hughson. 2002. Structural identification of a bacterial quorum-sensing signal containing boron. Nature 415:545-9.

9. Childers, B. M., X. Cao, G. G. Weber, B. Demeler, P. J. Hart, and K. E. Klose. 2011. N-terminal residues of the Vibrio cholerae virulence regulatory protein ToxT involved in dimerization and modulation by fatty acids. J Biol Chem 286:28644-55.

10. Cremers, C. M., and U. Jakob. 2013. Oxidant sensing by reversible disulfide bond formation. J Biol Chem 288:26489-96.

11. Cremers, C. M., D. Knoefler, V. Vitvitsky, R. Banerjee, and U. Jakob. 2014. Bile salts act as effective protein-unfolding agents and instigators of disulfide stress in vivo. Proc Natl Acad Sci U S A 111:E1610-9.

12. Dalia, A. B. 2018. Natural Cotransformation and Multiplex Genome Editing by Natural Transformation (MuGENT) of Vibrio cholerae. Methods Mol Biol 1839:5364.

13. Dalia, A. B., E. McDonough, and A. Camilli. 2014. Multiplex genome editing by natural transformation. Proc Natl Acad Sci U S A 111:8937-42.

14. Dawson, P. A. 1998. Bile secretion and the enterohepatic circulation of bile acids. In M. Feldman, M. H. Sleisenger, and B. F. Scharschmidt (ed.), Sleisenger and Fordtran's gastrointestinal and liver disease: pathophysiology/diagnosis/management, 6th ed. W. B. Saunders, Co., Philadelphia, PA.:1052-1063. 
15. Escobar, L. E., S. J. Ryan, A. M. Stewart-lbarra, J. L. Finkelstein, C. A. King, H. Qiao, and M. E. Polhemus. 2015. A global map of suitability for coastal Vibrio cholerae under current and future climate conditions. Acta Trop 149:202-11.

16. Falke, J. J., and D. E. Koshland, Jr. 1987. Global flexibility in a sensory receptor: a site-directed cross-linking approach. Science 237:1596-600.

17. Fong, J. C. N., K. A. Syed, K. E. Klose, and F. H. Yildiz. 2010. Role of Vibrio polysaccharide (vps) genes in VPS production, biofilm formation and Vibrio cholerae pathogenesis. Microbiology 156:2757-2769.

18. Fuchs, S., J. Pane-Farre, C. Kohler, M. Hecker, and S. Engelmann. 2007. Anaerobic gene expression in Staphylococcus aureus. J Bacteriol 189:4275-89.

19. Garcia-Quintanilla, M., A. I. Prieto, L. Barnes, F. Ramos-Morales, and J. Casadesus. 2006. Bile-induced curing of the virulence plasmid in Salmonella enterica serovar Typhimurium. J Bacteriol 188:7963-5.

20. Hammer, B. K., and B. L. Bassler. 2003. Quorum sensing controls biofilm formation in Vibrio cholerae. Mol Microbiol 50:101-4.

21. Higgins, D. A., M. E. Pomianek, C. M. Kraml, R. K. Taylor, M. F. Semmelhack, and B. L. Bassler. 2007. The major Vibrio cholerae autoinducer and its role in virulence factor production. Nature 450:883-6.

22. Huang, X., O. P. Duddy, J. E. Silpe, J. E. Paczkowski, J. Cong, B. R. Henke, and B. L. Bassler. 2020. Mechanism underlying autoinducer recognition in the Vibrio cholerae DPO-VqmA quorum-sensing pathway. J Biol Chem 295:29162931.

23. Hung, D. T., and J. J. Mekalanos. 2005. Bile acids induce cholera toxin expression in Vibrio cholerae in a ToxT-independent manner. Proc Natl Acad Sci U S A 102:3028-33.

24. Hung, D. T., J. Zhu, D. Sturtevant, and J. J. Mekalanos. 2006. Bile acids stimulate biofilm formation in Vibrio cholerae. Mol Microbiol 59:193-201.

25. Joelsson, A., B. Kan, and J. Zhu. 2007. Quorum sensing enhances the stress response in Vibrio cholerae. Appl Environ Microbiol 73:3742-6.

26. Johansson, M. E., H. Sjovall, and G. C. Hansson. 2013. The gastrointestinal mucus system in health and disease. Nat Rev Gastroenterol Hepatol 10:352-61.

27. Jung, S. A., C. A. Chapman, and W. L. Ng. 2015. Quadruple quorum-sensing inputs control Vibrio cholerae virulence and maintain system robustness. PLoS Pathog 11:e1004837.

28. Kelly, R. C., M. E. Bolitho, D. A. Higgins, W. Lu, W. L. Ng, P. D. Jeffrey, J. D. Rabinowitz, M. F. Semmelhack, F. M. Hughson, and B. L. Bassler. 2009. The Vibrio cholerae quorum-sensing autoinducer CAI-1: analysis of the biosynthetic enzyme CqsA. Nat Chem Biol 5:891-5.

29. Koestler, B. J., and C. M. Waters. 2014. Bile acids and bicarbonate inversely regulate intracellular cyclic di-GMP in Vibrio cholerae. Infect Immun 82:3002-14.

30. Kosower, N. S., and E. M. Kosower. 1995. Diamide: an oxidant probe for thiols. Methods Enzymol 251:123-33.

31. Krishnan, H. H., A. Ghosh, K. Paul, and R. Chowdhury. 2004. Effect of anaerobiosis on expression of virulence factors in Vibrio cholerae. Infect Immun 72:3961-7. 
32. Landeta, C., L. McPartland, N. Q. Tran, B. M. Meehan, Y. Zhang, Z. Tanweer, S. Wakabayashi, J. Rock, T. Kim, D. Balasubramanian, R. Audette, M. Toosky, J. Pinkham, E. J. Rubin, S. Lory, G. Pier, D. Boyd, and J. Beckwith. 2019. Inhibition of Pseudomonas aeruginosa and Mycobacterium tuberculosis disulfide bond forming enzymes. Mol Microbiol 111:918-937.

33. Lee, K. M., Y. Park, W. Bari, M. Y. Yoon, J. Go, S. C. Kim, H. I. Lee, and S. S. Yoon. 2012. Activation of cholera toxin production by anaerobic respiration of trimethylamine $\mathrm{N}$-oxide in Vibrio cholerae. J Biol Chem 287:39742-52.

34. Liu, Z., A. Hsiao, A. Joelsson, and J. Zhu. 2006. The transcriptional regulator VqmA increases expression of the quorum-sensing activator HapR in Vibrio cholerae. J Bacteriol 188:2446-53.

35. Liu, Z., M. Yang, G. L. Peterfreund, A. M. Tsou, N. Selamoglu, F. Daldal, Z. Zhong, B. Kan, and J. Zhu. 2010. Vibrio cholerae anaerobic induction of virulence gene expression is controlled by thiol-based switches of virulence regulator AphB. Proc Natl Acad Sci U S A 108:810-5.

36. Lowden, M. J., K. Skorupski, M. Pellegrini, M. G. Chiorazzo, R. K. Taylor, and F. J. Kull. 2010. Structure of Vibrio cholerae ToxT reveals a mechanism for fatty acid regulation of virulence genes. Proc Natl Acad Sci U S A 107:2860-5.

37. Mashruwala, A. A., S. Bhatt, S. Poudel, E. S. Boyd, and J. M. Boyd. 2016. The DUF59 Containing Protein SufT Is Involved in the Maturation of Iron-Sulfur (FeS) Proteins during Conditions of High FeS Cofactor Demand in Staphylococcus aureus. PLoS Genet 12:e1006233.

38. Masuda, S., C. Dong, D. Swem, A. T. Setterdahl, D. B. Knaff, and C. E. Bauer. 2002. Repression of photosynthesis gene expression by formation of a disulfide bond in CrtJ. Proc Natl Acad Sci U S A 99:7078-83.

39. Miller, M. B., K. Skorupski, D. H. Lenz, R. K. Taylor, and B. L. Bassler. 2002. Parallel quorum sensing systems converge to regulate virulence in Vibrio cholerae. Cell 110:303-14.

40. Muras, V., P. Dogaru-Kinn, Y. Minato, C. C. Hase, and J. Steuber. 2016. The $\mathrm{Na}+-$ Translocating NADH:Quinone Oxidoreductase Enhances Oxidative Stress in the Cytoplasm of Vibrio cholerae. J Bacteriol 198:2307-17.

41. Nozaki, S., and H. Niki. 2019. Exonuclease III (XthA) Enforces In Vivo DNA Cloning of Escherichia coli To Create Cohesive Ends. J Bacteriol 201.

42. Oktiabrskii, O. N., Smirnova, G. V. 2012. Redox potential changes in bacterial cultures under stress conditions. Microbiology 81:131-142.

43. Olive, A. J., R. Kenjale, M. Espina, D. S. Moore, W. L. Picking, and W. D. Picking. 2007. Bile salts stimulate recruitment of IpaB to the Shigella flexneri surface, where it colocalizes with IpaD at the tip of the type III secretion needle. Infect Immun 75:2626-9.

44. Ottemann, K. M., and J. J. Mekalanos. 1996. The ToxR protein of Vibrio cholerae forms homodimers and heterodimers. J Bacteriol 178:156-62.

45. Papenfort, K., and B. L. Bassler. 2016. Quorum sensing signal-response systems in Gram-negative bacteria. Nat Rev Microbiol 14:576-88.

46. Papenfort, K., K. U. Forstner, J. P. Cong, C. M. Sharma, and B. L. Bassler. 2015. Differential RNA-seq of Vibrio cholerae identifies the VqmR small RNA as a regulator of biofilm formation. Proc Natl Acad Sci U S A 112:E766-75. 
47. Papenfort, K., J. E. Silpe, K. R. Schramma, J. P. Cong, M. R. Seyedsayamdost, and B. L. Bassler. 2017. A Vibrio cholerae autoinducerreceptor pair that controls biofilm formation. Nat Chem Biol 13:551-557.

48. Parashar, V., C. Aggarwal, M. J. Federle, and M. B. Neiditch. 2015. Rgg protein structure-function and inhibition by cyclic peptide compounds. Proc Natl Acad Sci U S A 112:5177-82.

49. Pereira, C. S., J. A. Thompson, and K. B. Xavier. 2013. Al-2-mediated signalling in bacteria. FEMS Microbiol Rev 37:156-81.

50. Plecha, S. C., and J. H. Withey. 2015. Mechanism for inhibition of Vibrio cholerae ToxT activity by the unsaturated fatty acid components of bile. J Bacteriol 197:1716-25.

51. Repetto, G., A. del Peso, and J. L. Zurita. 2008. Neutral red uptake assay for the estimation of cell viability/cytotoxicity. Nat Protoc 3:1125-31.

52. Rossius, M., F. Hochgrafe, and H. Antelmann. 2018. Thiol-Redox Proteomics to Study Reversible Protein Thiol Oxidations in Bacteria. Methods Mol Biol 1841:261-275.

53. Schauder, S., and B. L. Bassler. 2001. The languages of bacteria. Genes Dev 15:1468-80.

54. Schauder, S., K. Shokat, M. G. Surette, and B. L. Bassler. 2001. The LuxS family of bacterial autoinducers: biosynthesis of a novel quorum-sensing signal molecule. Mol Microbiol 41:463-76.

55. Scheele, G., and R. Jacoby. 1982. Conformational changes associated with proteolytic processing of presecretory proteins allow glutathione-catalyzed formation of native disulfide bonds. J Biol Chem 257:12277-82.

56. Suckow, G., P. Seitz, and M. Blokesch. 2011. Quorum sensing contributes to natural transformation of Vibrio cholerae in a species-specific manner. J Bacteriol 193:4914-24.

57. Taylor, R. K., V. L. Miller, D. B. Furlong, and J. J. Mekalanos. 1987. Use of phoA gene fusions to identify a pilus colonization factor coordinately regulated with cholera toxin. Proc Natl Acad Sci U S A 84:2833-7.

58. Trotter, E. W., and C. M. Grant. 2002. Thioredoxins are required for protection against a reductive stress in the yeast Saccharomyces cerevisiae. Mol Microbiol 46:869-78.

59. Waters, C. M., and B. L. Bassler. 2005. Quorum sensing: cell-to-cell communication in bacteria. Annu Rev Cell Dev Biol 21:319-46.

60. Weber, G. G., and K. E. Klose. 2011. The complexity of ToxT-dependent transcription in Vibrio cholerae. Indian J Med Res 133:201-6.

61. Winzer, K., K. R. Hardie, N. Burgess, N. Doherty, D. Kirke, M. T. G. Holden, R. Linforth, K. A. Cornell, A. J. Taylor, P. J. Hill, and P. Williams. 2002. LuxS: its role in central metabolism and the in vitro synthesis of 4-hydroxy-5-methyl3(2H)-furanone. Microbiology 148:909-922.

62. Xue, Y., F. Tu, M. Shi, C. Q. Wu, G. Ren, X. Wang, W. Fang, H. Song, and M. Yang. 2016. Redox pathway sensing bile salts activates virulence gene expression in Vibrio cholerae. Mol Microbiol 102:909-924.

63. Yang, M., Z. Liu, C. Hughes, A. M. Stern, H. Wang, Z. Zhong, B. Kan, W. Fenical, and J. Zhu. 2013. Bile salt-induced intermolecular disulfide bond 
formation activates Vibrio cholerae virulence. Proc Natl Acad Sci U S A 110:2348-53.

64. Zheng, L., C. J. Kelly, and S. P. Colgan. 2015. Physiologic hypoxia and oxygen homeostasis in the healthy intestine. A Review in the Theme: Cellular Responses to Hypoxia. Am J Physiol Cell Physiol 309:C350-60.

1051

1052

1053

65. Zheng, M., F. Aslund, and G. Storz. 1998. Activation of the OxyR transcription factor by reversible disulfide bond formation. Science 279:1718-21.

66. Zhu, J., and J. J. Mekalanos. 2003. Quorum sensing-dependent biofilms enhance colonization in Vibrio cholerae. Dev Cell 5:647-56.

67. Zhu, J., M. B. Miller, R. E. Vance, M. Dziejman, B. L. Bassler, and J. J. Mekalanos. 2002. Quorum-sensing regulators control virulence gene expression in Vibrio cholerae. Proc Natl Acad Sci U S A 99:3129-34. 